\title{
Phloroglucinol Strengthens the Antioxidant Barrier and Reduces Oxidative/Nitrosative Stress in Nonalcoholic Fatty Liver Disease (NAFLD)
}

\author{
Krzysztof Drygalski $\mathbb{D}$, ${ }^{1,2}$ Katarzyna Siewko ${ }^{D},{ }^{2}$ Andrzej Chomentowski, ${ }^{3}$ \\ Cezary Odrzygóźdź, ${ }^{4}$ Anna Zalewska ${ }^{D},{ }^{5}$ Adam Krętowski $\mathbb{D}^{1,2}$ and Mateusz Maciejczyk ${ }^{1}{ }^{6}$ \\ ${ }^{1}$ Clinical Research Center, Medical University of Bialystok, Poland \\ ${ }^{2}$ Department of Endocrinology, Diabetology and Internal Medicine, Medical University of Bialystok, Poland \\ ${ }^{3}$ Department of Biophysics, Medical University of Bialystok, Poland \\ ${ }^{4}$ Department of Molecular and Systems Biology, Institute of Bioorganic Chemistry, Polish Academy of Sciences, Poznań, Poland \\ ${ }^{5}$ Experimental Dentistry Laboratory, Medical University of Bialystok, Poland \\ ${ }^{6}$ Department of Hygiene, Epidemiology and Ergonomics, Medical University of Bialystok, Poland
}

Correspondence should be addressed to Mateusz Maciejczyk; mat.maciejczyk@gmail.com

Received 25 August 2020; Revised 12 December 2020; Accepted 31 December 2020; Published 15 January 2021

Academic Editor: Ayman Mahmoud

Copyright (c) 2021 Krzysztof Drygalski et al. This is an open access article distributed under the Creative Commons Attribution License, which permits unrestricted use, distribution, and reproduction in any medium, provided the original work is properly cited.

\begin{abstract}
Nonalcoholic fatty liver disease (NAFLD) is one of the most commonly occurring diseases within western dietary patterns. Usually untreated, it may lead to type 2 diabetes mellitus (T2DM), steatohepatitis (NASH), and hepatocellular carcinoma (HCC). Besides its severe aftermath, up to now, there is no known therapeutic approach to this disease in everyday clinical practice. Most NAFLD patients are encouraged to do physical activities or diet change and remain without pharmacological treatment. In this study, we present phloroglucinol (PHG) as a novel and promising compound in NAFLD treatment. PHG significantly increased the level of enzymatic and nonenzymatic antioxidants both in palmitate and hydrogen peroxide-induced oxidative stress models. Strengthened antioxidative defense reduced the oxidative/nitrosative damage to cell proteins, lipids, and carbohydrates. Furthermore, PHG treatment reduced hepatic steatosis; lowered inflammatory markers, such as NF- $\kappa$ B or HIF- $1 \alpha$; and inhibited cell apoptosis. Moreover, PHG had a more comprehensive effect than other commonly used antioxidants: N-acetylcysteine (NAC) and $\alpha$-lipoic acid (ALA), suggesting its clinical usability. Therefore, our paper supports the benefits of natural compounds as a therapeutical approach to NAFLD.
\end{abstract}

\section{Introduction}

Nonalcoholic fatty liver disease (NAFLD) is a chronic medical condition associated with the excessive accumulation of free fatty acids, diglycerides, and triglycerides in the liver [1]. NAFLD's leading cause has been attributed to excessive high fat intake referred to as "western dietary pattern." It is estimated that around $25 \%$ of the world's population might be affected, whereas, in western countries, prevalence is higher [2]. The lipid overload state present in NAFLD results in a dysregulation of hepatocytes' metabolic activity, leading to inflammatory response and liver tissue injury [3]. The most common aftermath of liver steatosis in NAFLD is insulin resistance, leading to type 2 diabetes mellitus (T2DM) [46]. Additionally, patients affected by NAFLD are also more likely to develop systemic hypertension, while frequent vascular complications increase the rate of cardiac incidents [7-9]. What is more, long-lasting steatosis induces oxidative stress and inflammation which results in the development of nonalcoholic steatohepatitis (NASH). It remains the leading cause of hepatocellular carcinoma (HCC) in western countries [1-3].

Oxidative stress plays a critical role in the progression of NAFLD $[10,11]$. Generally, oxidative stress refers to the 
imbalance between the formation of oxygen/nitrogen free radicals and the efficiency of mechanisms responsible for their elimination. Under physiological conditions, reactive oxygen species (ROS) formed in metabolic processes are effectively scavenged by enzymatic and nonenzymatic antioxidants. However, under pathology, redox imbalance occurs in favor of oxidation reactions, and as a consequence, overproduction of ROS causes oxidative damage to biomolecules and cell structures [12]. The oxidative damage may manifest as both protein and lipid oxidation and the creation of advanced glycation end products (AGEs). Increased production of ROS in NAFLD relates to blockage of $\beta$-oxidation, which triggers an impairment of the mitochondrial electron transport chain [13]. What is more, the incomplete oxidation of acyl-carnitine causes aggregation of lipotoxic intermediates, which can be an indirect source of ROS [14]. Additionally, recent studies showed the possible role of cytochrome CYP2E1 as a source of ROS in the liver tissue [15]. Since there is no approved medication for NAFLD, whereas mild to moderate lifestyle changes do not bring significant benefits to NAFLD patients, it is crucial to investigate potentially effective drug treatment [16]. Current therapeutic approaches focus their efforts on the reduction of oxidative stress. As for now, silymarin, vitamins $\mathrm{E}$ and $\mathrm{D}$, polyunsaturated fatty acids of the omega-3 series, coenzyme Q10, berberine, and curcumin are considered to exert moderate effects after prolonged use [17]. Out of the presented substances, the effects of vitamin E seem to be best documented. However, there are some concerns about the safety of vitamin E supplementation. It has been reported that oral vitamin E supplementation among healthy men increases prostate cancer risk and risk for hemorrhagic stroke in the general population and heart failure in patients with left ventricular dysfunction [18-20]. Based on the above information, we chose phloroglucinol (PHG), a phenolic compound of natural origin, mainly known for its nonspecific antispasmodic properties in gastric tract disorders. Phloroglucinol is safe to use and constitutes an active ingredient in various antispasmodic compositions [21]. Indeed, it has been proven that PHG may have anti-inflammatory and antioxidant capabilities in different medical conditions [22, 23]. Some studies even report its anticancer potential $[24,25]$. Nevertheless, it has never been assessed as a potential drug counteracting NAFLD and preventing its progress towards NASH.

In our study, we developed a HepG2 cell line to assess their response to the changes in oxidative balance caused by NAFLD and the feasibility of alleviating these effects using PHG. Furthermore, we compared the effectiveness of PHG with two other compounds of renowned antioxidative properties: $\mathrm{N}$-acetylcysteine (NAC) and $\alpha$-lipoic acid (ALA). The oxidative stress was generated in two models: hydrogen peroxide or palmitic acid-induced steatosis.

\section{Results}

2.1. Cell Viability. PHG showed a dose-dependent decrease of cell viability which was statistically significant in concentrations above $100 \mu \mathrm{M}(200 \mu \mathrm{M}$ : $-33.6 \% p<0.05 ; 400 \mu \mathrm{M}$ : $-47.3 \% p<0.01 ; 1000 \mu \mathrm{M}:-82.7 \% p<0.001) . \mathrm{H}_{2} \mathrm{O}_{2}$ also decreased cell viability in a dose-dependent manner, which was significant in concentrations above $1 \mathrm{mM}(2 \mathrm{mM}$ : $-20.4 \% p<0.01 ; 5 \mathrm{mM}:-35.8 \% p<0.0001 ; 10 \mathrm{mM}:-59.7 \% p$ $<0.0001)$. With the addition of PHG to $10 \mathrm{mM} \mathrm{H}_{2} \mathrm{O}_{2}$, the medium did not affect cell viability in concentrations below $200 \mu \mathrm{M}$ of PHG. Basing on the cell viability, we choose the PHG concentration of $100 \mu \mathrm{M}$ and $10 \mathrm{mM}$ concentration of $\mathrm{H}_{2} \mathrm{O}_{2}$ for further experiments (Figure 1).

2.2. Antioxidant Defense. Antioxidants are substances that, in low concentrations, protect against oxidation or delay the oxidation of cell components. In our study, we used both enzymatic (catalase, CAT; glutathione peroxidase, GSH-Px; glutathione reductase, GR; superoxide dismutase, SOD) and nonenzymatic (reduced glutathione, GSH) antioxidants to assess the antioxidant barrier.

2.2.1. NAFLD Model. PHG lowered cellular total glutathione content (PHG: $-25.1 \% p<0.05$ ) and GSH concentration (PHG: $-45.4 \%$; NAFLD: $-65.7 \% p<0.0001$; NAFLD+PHG: $-18.8 \%$, NAFLD+NAC: $+0.9 \%$, NAFLD+ALA: $+10.3 \%)$. All the analyzed antioxidants decreased PA-induced GSSG (NAFLD:+38.8\% $p<0.01$; NAFLD+PHG: $-3.9 \% p<0.01$; NAFLD+NAC: $-14 \% p<0.001$; NAFLD+ALA: $-19.9 \% p<$ 0.0001 ), and some of them normalised the GSH/GSSG ratio (NAFLD+NAC: $-23.6 \% p<0.05$; NAFLD+ALA: $-59.3 \% p<$ $0.001)$. Moreover, the activity of GSH-Px was markedly elevated in the NAFLD+PHG-treated group while being significantly lower in the group treated with NAFLD alone (PHG:10.3\%; NAFLD: $-57.2 \% p<0.0001$; NAFLD+PHG: $+33.5 \%$ $p<0.001$; NAFLD+NAC: $-5.8 \%$; NAFLD+ALA: $+15.5 \%)$. The activity of CAT was increased in all but one (PHG) of the experimental groups (PHG: $+35.1 \%$; NAFLD: $+171.7 \%$ $p<0.01$; NAFLD+PHG: $+364 \% p<0.0001$; NAFLD+NAC: $+244.7 \% p<0.0001$; NAFLD+ALA: $+246.4 \% p<0.0001$ ) when compared to the control and significantly higher in NAFLD+PHG when compared to other groups. Additionally, PHG together with NAFLD elevated GR activity compared to all groups except NAFLD+ALA (PHG: +6.9\%; NAFLD: $\quad-19.9 \%$; NAFLD+PHG: $\quad+57.1 \% \quad p<0.0001$; NAFLD+NAC: $+13.7 \%$, NAFLD+ALA: $+26.3 \%)$. PHG had no substantial impact on SOD activity (PHG: $-12 \%$; NAFLD: $-64.5 \% p<0.0001$; NAFLD+PHG: $+15.2 \%$, NAFLD+NAC: $-18.2 \%$, NAFLD+ALA:-7.2\%) (Figure 2).

2.2.2. $\mathrm{H}_{2} \mathrm{O}_{2}$ Model. Total glutathione was slightly decreased in the $\mathrm{H}_{2} \mathrm{O}_{2}+$ PHG group $(-27.8 \% p<0.05)$. Both PHG and $\mathrm{H}_{2} \mathrm{O}_{2}$ alone depleted GSH with no significant differences to control in other groups (PHG: $-45.3 \% p<0.01 ; \mathrm{H}_{2} \mathrm{O}_{2}:-70 \%$ $p<0.0001)$ However, NAC and ALA normalised $\mathrm{H}_{2} \mathrm{O}_{2}$ induced GSH drop $\left(\mathrm{H}_{2} \mathrm{O}_{2}+\right.$ NAC: $-5.5 \% ; \mathrm{H}_{2} \mathrm{O}_{2}+$ ALA: $-3.3 \%$ $p<0.0001)$. In contrast, $\mathrm{H}_{2} \mathrm{O}_{2}$ decreased GSH-Px activity with nonsignificant elevation in the group treated with $\mathrm{H}_{2} \mathrm{O}_{2}+$ PHG (PHG: $-10.3 \% ; \mathrm{H}_{2} \mathrm{O}_{2}:-55.8 \% p<0.001 ; \mathrm{H}_{2} \mathrm{O}_{2}$ +PHG: +30.7\%; $\mathrm{H}_{2} \mathrm{O}_{2}+\mathrm{NAC}:+2.6 \% ; \mathrm{H}_{2} \mathrm{O}_{2}$ +ALA: +15.4\%). $\mathrm{H}_{2} \mathrm{O}_{2}$ significantly increased the GSSG concentration which was normalised by all analyzed antioxidants $\left(\mathrm{H}_{2} \mathrm{O}_{2}:+52.8 \%\right.$ $p<0.001 ; \mathrm{H}_{2} \mathrm{O}_{2}+$ PHG: $-18.7 \% ; \mathrm{H}_{2} \mathrm{O}_{2}+\mathrm{NAC}:-37.9 \% ; \mathrm{H}_{2} \mathrm{O}_{2}$ +ALA: $-13.5 \% p<0.0001)$. GSH/GSSG ratio was decreased 


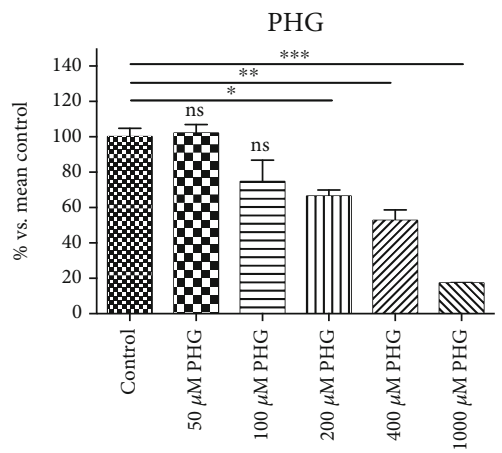

(a)

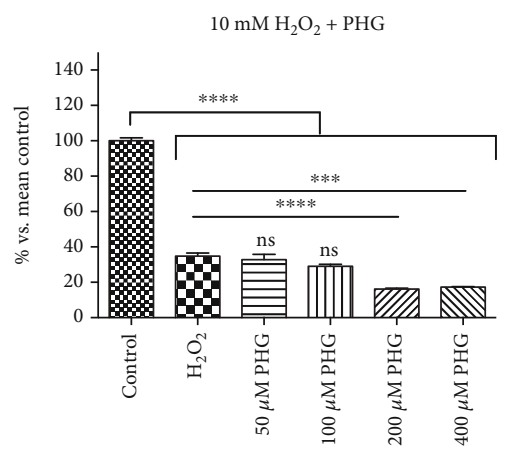

(c)

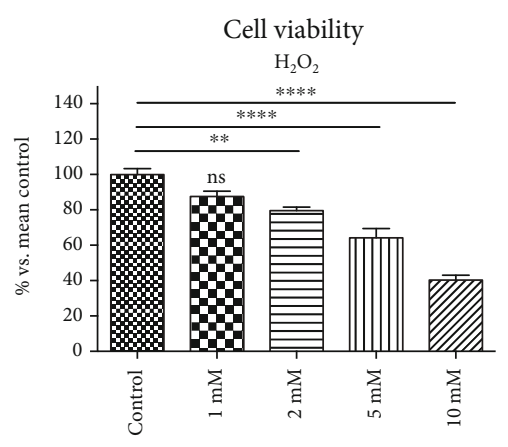

(b)

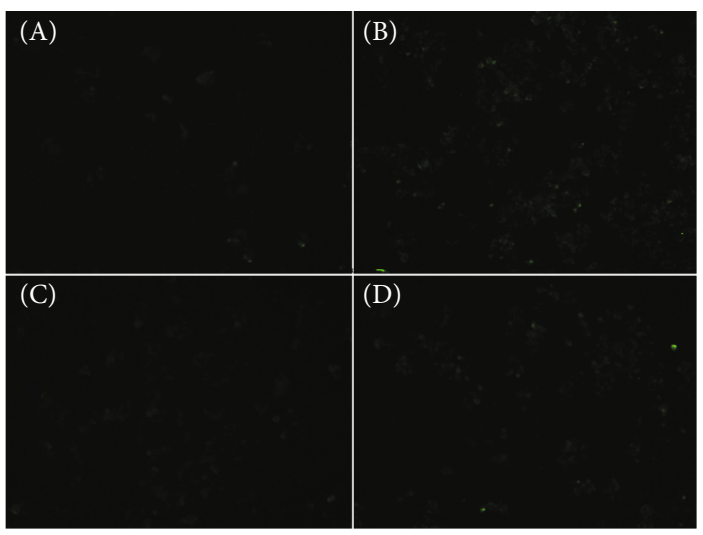

(d)

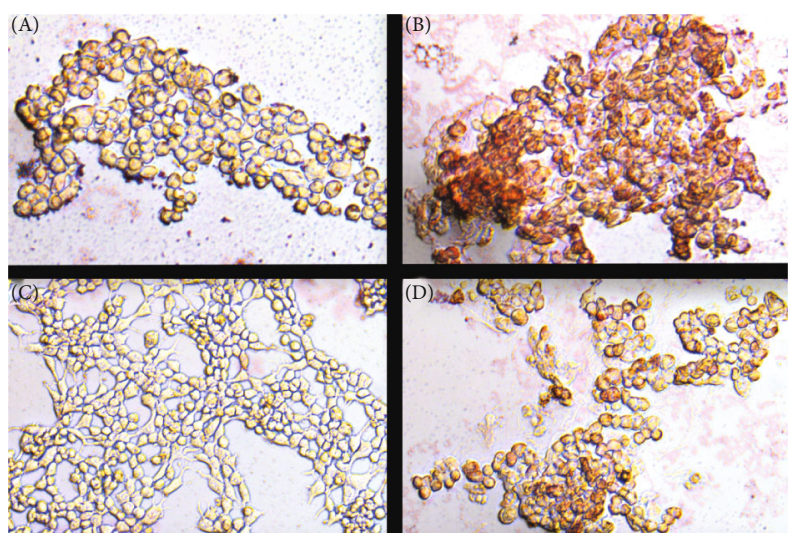

(e)

Figure 1: The effect of PHG on cell viability and steatosis. (a, c) PHG was nontoxic, both referring to the control and $\mathrm{H}_{2} \mathrm{O}_{2}$ in concentrations below $200 \mu \mathrm{M}$ and dose-dependently lowered cell viability in concentrations above $200 \mu \mathrm{M}$. (b) $\mathrm{H}_{2} \mathrm{O}_{2}$ showed a dose-dependent effect on cell viability with $\mathrm{GI}_{50}$ around $10 \mathrm{mM}$. (d) Immunofluorescence staining of active Caspase 3 (A: control group; B: NAFLD; C: PHG; D: NAFLD + PHG). (e) Oil Red O staining of HepG2 cells (A: control group; B: NAFLD; C: PHG; D: NAFLD+PHG).

by $\mathrm{H}_{2} \mathrm{O}_{2}$ and normalised by NAC and ALA $\left(\mathrm{H}_{2} \mathrm{O}_{2}:-94.1 \%\right.$ $p<0.05 ; \mathrm{H}_{2} \mathrm{O}_{2}+\mathrm{NAC}:+58.3 \% p<0.0001 ; \mathrm{H}_{2} \mathrm{O}_{2}+\mathrm{ALA}:+6 \%$ $p<0.01)$. The $\mathrm{H}_{2} \mathrm{O}_{2}+\mathrm{PHG}$ group generated a rise of $\mathrm{GR}$ activity, in opposition to the group with $\mathrm{H}_{2} \mathrm{O}_{2}$ only, in which we noted low activity of GR (PHG: $+6.9 \% ; \mathrm{H}_{2} \mathrm{O}_{2}:-43.1 \% p$ $<0.05 ; \mathrm{H}_{2} \mathrm{O}_{2}+\mathrm{PHG}:+61.7 \% p<0.01 ; \mathrm{H}_{2} \mathrm{O}_{2}+\mathrm{NAC}:+2.1 \%$; $\mathrm{H}_{2} \mathrm{O}_{2}+$ ALA: $+38.7 \%$ ). CAT activity was significantly increased only by incubation with $\mathrm{H}_{2} \mathrm{O}_{2}$ (PHG: $+35.1 \%$; $\mathrm{H}_{2} \mathrm{O}_{2}$ : +61\% $p<0.05 ; \mathrm{H}_{2} \mathrm{O}_{2}+\mathrm{PHG}:+30.6 \% ; \mathrm{H}_{2} \mathrm{O}_{2}+\mathrm{NAC}$ : $\left.+54.5 \% ; \mathrm{H}_{2} \mathrm{O}_{2}+\mathrm{ALA}:+49.3 \%\right)$. PHG, NAC, and ALA together with $\mathrm{H}_{2} \mathrm{O}_{2}$ decreased the SOD level (PHG: $-12 \%$;
$\mathrm{H}_{2} \mathrm{O}_{2}:-2 \% ; \mathrm{H}_{2} \mathrm{O}_{2}:+$ PHG: $-31.4 \% p<0.01 ; \mathrm{H}_{2} \mathrm{O}_{2}+\mathrm{NAC}$ : $-38 \% \quad p<0.0001 ; \quad \mathrm{H}_{2} \mathrm{O}_{2}+$ ALA: $\left.\quad-46.1 \% \quad p<0.0001\right)$ (Figure 3).

2.3. ROS Production and Nitrosative Stress. For the evaluation of ROS production rate, we determined the NADPH oxidase activity (NOX), which is the main prooxidative enzyme responsible for the formation of free radicals in the cell. For the assessment of nitrosative stress, we used both nitric oxide (NO) and peroxynitrite (the most reactive form of RNS (reactive nitrogen species)). 


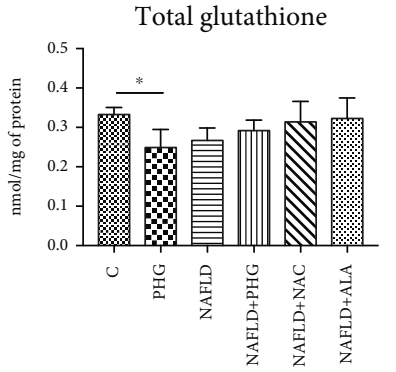

(a)

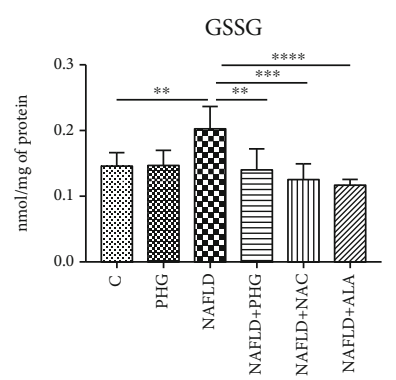

(c)

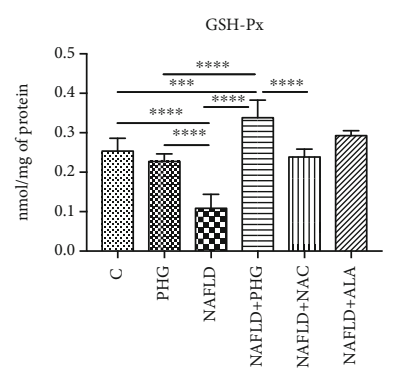

(e)

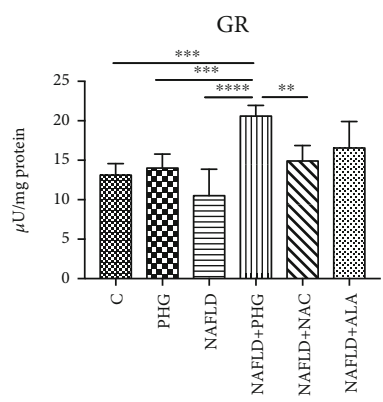

(g)

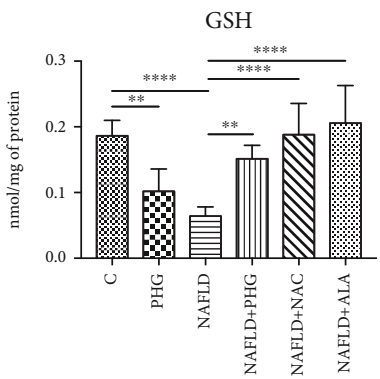

(b)

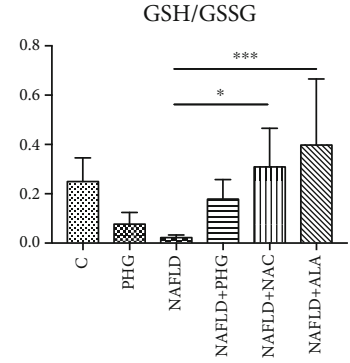

(d)

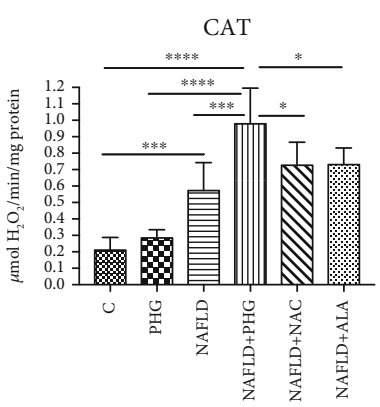

(f)

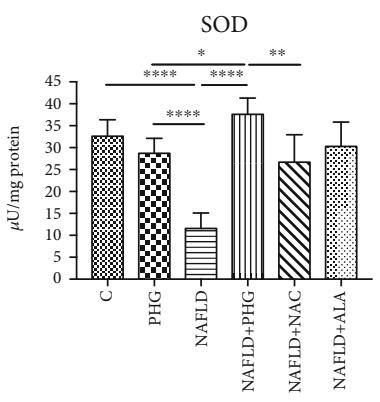

(h)

FIgURE 2: Antioxidant defense in the NAFLD model. The activity of the enzymatic and nonenzymatic antioxidants in HepG2 cell cultures incubated with phloroglucinol (PHG), palmitate (NAFLD), both PHG and NAFLD, and palmitate with other known antioxidants: Nacetylcysteine (NAFLD+NAC) and alpha-lipoic acid (NAFLD+ALA). (a) Phloroglucinol alone decreased the concentration of total glutathione; however, no effect was observed in the group incubated with palmitate and PHG together. (b-d) PHG similarly to other antioxidants normalised the level of reduced and oxidized glutathione but not the GSH/GSSG ratio. (e) The intensity of glutathione peroxidase activity (GSH-Px) was markedly elevated in the NAFLD+PHG group. (f) The activity of catalase (CAT) was significantly higher in the NAFLD+PHG group compared to other groups. (g) PHG increased the activity of glutathione reductase (GR) in lipid overload state (NAFLD+PHG). (h) PHG exerted no effect on superoxide dismutase compared with the control; however, the difference was significant between the NAFLD, PHG, and NAFLD+PHG groups.

2.3.1. NAFLD Model. Both NOX and peroxynitrite were markedly elevated in the NAFLD group (PHG: $-26.2 \%$; NAFLD: $+103.6 \% \quad p<0.0001$; NAFLD+PHG: $+13.8 \%$; NAFLD+NAC: $-5.4 \%$; NAFLD+ALA: $-3.1 \%$ ) (PHG: $+22 \%$;
NAFLD: $\quad+98.3 \% \quad p<0.0001 ; \quad$ NAFLD+PHG: $+39.4 \%$; NAFLD+NAC: $+14.2 \%$; NAFLD+ALA: $+17.4 \%)$ as compared to the control. NO level was significantly lower in the NAFLD and NAFLD+PHG groups (PHG: $-4.3 \%$; NAFLD: 


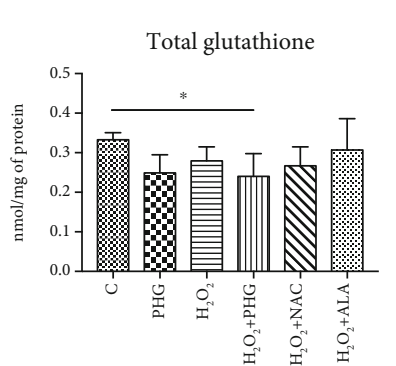

(a)

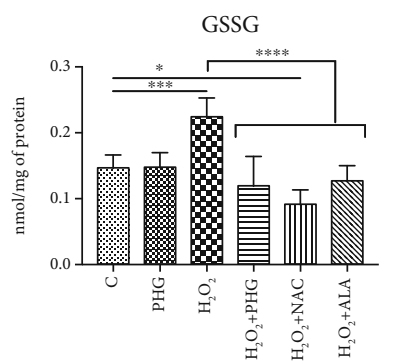

(c)

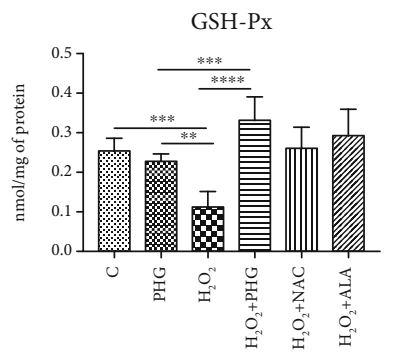

(e)

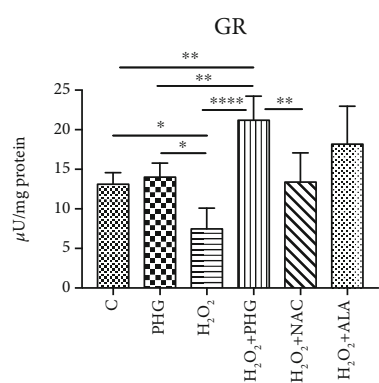

(g)

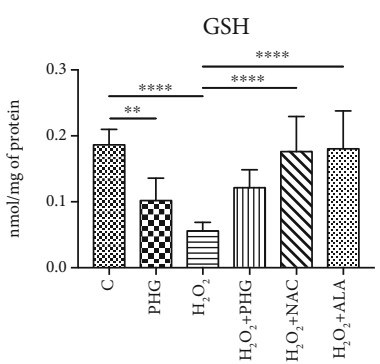

(b)

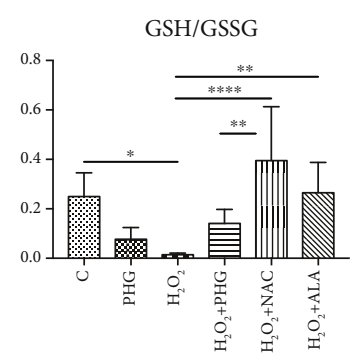

(d)

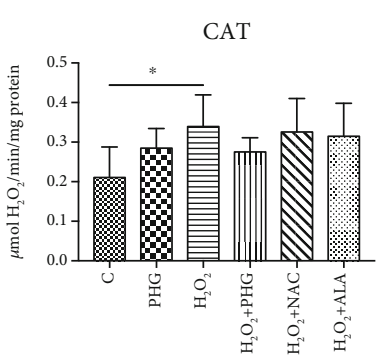

(f)

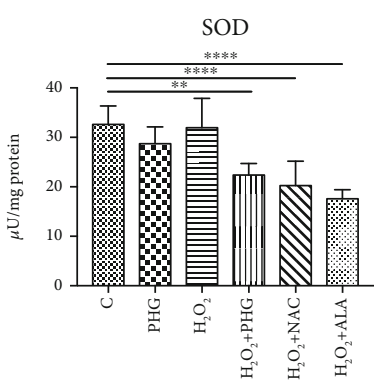

(h)

Figure 3: Antioxidant defense in the $\mathrm{H}_{2} \mathrm{O}_{2}$ model. The activity of the enzymatic and nonenzymatic antioxidants in HepG2 cell cultures incubated with phloroglucinol (PHG), hydrogen peroxide $\left(\mathrm{H}_{2} \mathrm{O}_{2}\right)$, both $\mathrm{PHG}$ and $\mathrm{H}_{2} \mathrm{O}_{2}$, and hydrogen peroxide with other known antioxidants: $\mathrm{N}$-acetylcysteine $\left(\mathrm{H}_{2} \mathrm{O}_{2}+\mathrm{NAC}\right)$ alpha-lipoic acid $\left(\mathrm{H}_{2} \mathrm{O}_{2}+\mathrm{ALA}\right)$. (a) The concentration of total glutathione (GSH) was slightly lowered only in the $\mathrm{H}_{2} \mathrm{O}_{2}+\mathrm{PHG}$ group. (b-d) PHG similar to other antioxidants normalised the level of reduced and oxidized glutathione but not the GSH/GSSG ratio. (e,f) Phloroglucinol significantly increased the activity of glutathione peroxidase (GSH-Px) decreased by $\mathrm{H}_{2} \mathrm{O}_{2}$. The exposure to $\mathrm{H}_{2} \mathrm{O}_{2}$ stimulated the activity of catalase (CAT). (g) The activity of glutathione reductase (GR) was intensified by phloroglucinol in the NAFLD+PHG group. (h) Phloroglucinol and other antioxidants decreased the activity of superoxide dismutase in NAFLD conditions.

$-46.7 \% \quad p<0.0001 ; \quad$ NAFLD+PHG: $\quad-37.5 \% \quad p<0.001)$ (Figure 4).

2.3.2. $\mathrm{H}_{2} \mathrm{O}_{2}$ Model. Incubation with $\mathrm{H}_{2} \mathrm{O}_{2}$ resulted in substantially increased creation of peroxynitrite (PHG: $-26.2 \%$; $\mathrm{H}_{2} \mathrm{O}_{2}:+161.2 \% p<0.0001 ; \mathrm{H}_{2} \mathrm{O}_{2}+\mathrm{PHG}:+40.7 \% p<0.01$; $\mathrm{H}_{2} \mathrm{O}_{2}+\mathrm{NAC}:+27 \% ; \mathrm{H}_{2} \mathrm{O}_{2}+$ ALA: $\left.+10.5 \%\right)$ as well as elevated activity of NOX (PHG: $+22 \% ; \mathrm{H}_{2} \mathrm{O}_{2}:+150.6 \% p<0.0001$;
$\mathrm{H}_{2} \mathrm{O}_{2}+$ PHG: $+67.8 \% ; \mathrm{H}_{2} \mathrm{O}_{2}+\mathrm{NAC}:+48.8 \% ; \mathrm{H}_{2} \mathrm{O}_{2}+$ ALA: $+36.7 \%)$. Concentration of $\mathrm{NO}$ was lowered in groups incubated with $\mathrm{H}_{2} \mathrm{O}_{2}, \mathrm{H}_{2} \mathrm{O}_{2}+\mathrm{PHG}$, and $\mathrm{H}_{2} \mathrm{O}_{2}+\mathrm{NAC}$ (PHG: $-4.3 \% ; \mathrm{H}_{2} \mathrm{O}_{2}:-28.3 \% p<0.05 ; \mathrm{H}_{2} \mathrm{O}_{2}+$ PHG: $-30.8 \% p<0.01$; $\mathrm{H}_{2} \mathrm{O}_{2}+\mathrm{NAC}-36.8 \% p<0.001$ ) (Figure 4 ).

2.4. Protein Glycooxidative Damage. For the evaluation of protein glycooxidation products, we used oxidative modified 


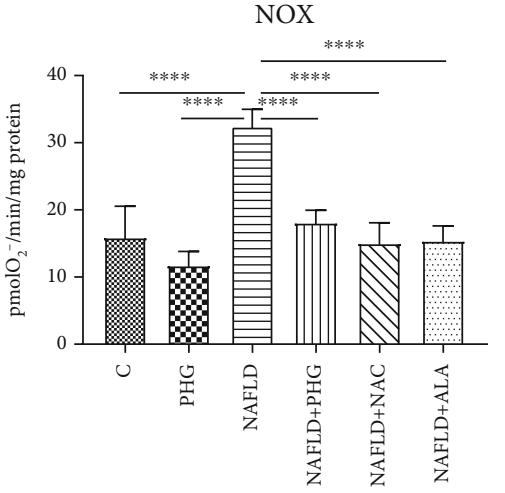

(a)

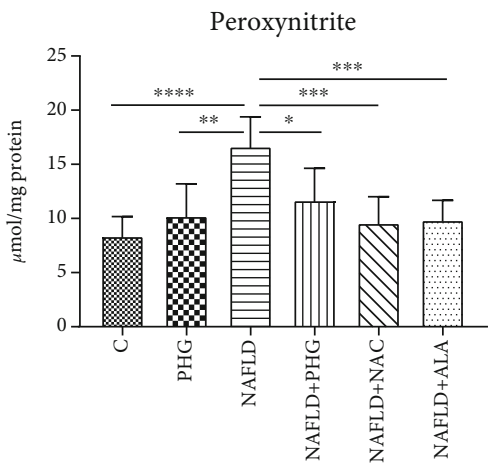

(c)

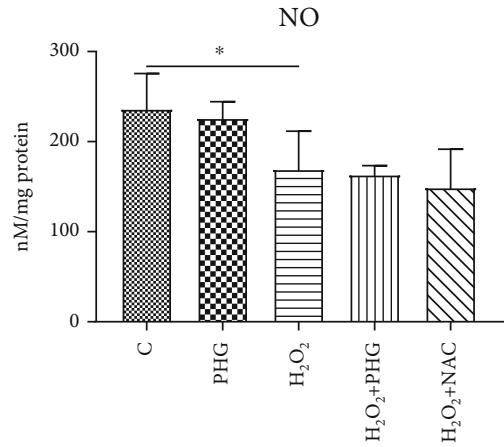

(e)

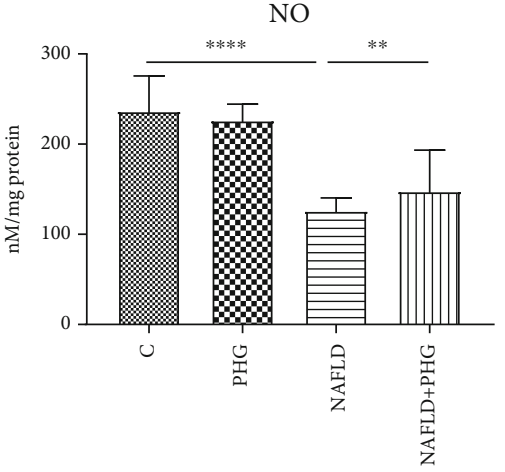

(b)

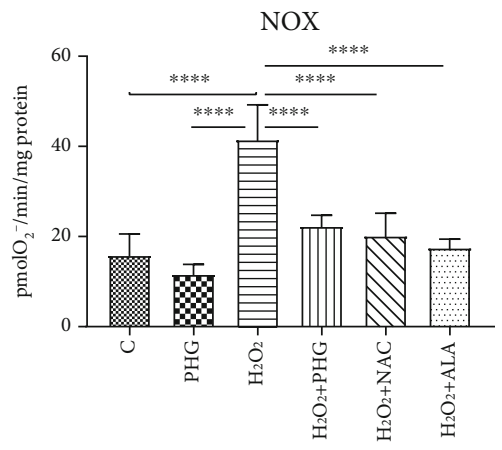

(d)

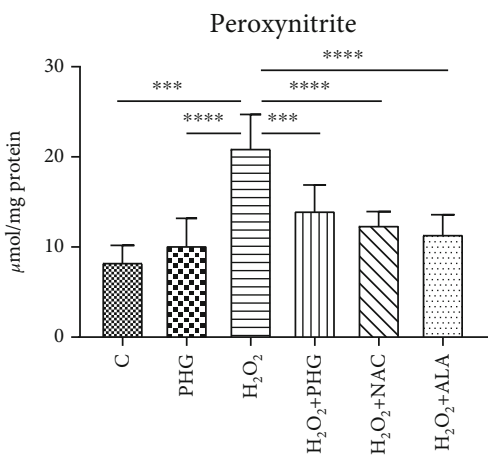

(f)

FIgURE 4: Concentration of the nitrosative stress products in HepG2. Cell cultures incubated with (first row) phloroglucinol (PHG), palmitate (NAFLD), both PHG and NAFLD, or palmitate with other known antioxidants: N-acetylcysteine (NAFLD+NAC) and alpha-lipoic acid (NAFLD+ALA); (second row) phloroglucinol (PHG), hydrogen peroxide $\left(\mathrm{H}_{2} \mathrm{O}_{2}\right)$, both $\mathrm{PHG}$ and $\mathrm{H}_{2} \mathrm{O}_{2}$, and hydrogen peroxide with other known antioxidants: $\mathrm{N}$-acetylcysteine $\left(\mathrm{H}_{2} \mathrm{O}_{2}+\mathrm{NAC}\right)$ alpha-lipoic acid $\left(\mathrm{H}_{2} \mathrm{O}_{2}+\mathrm{ALA}\right)$. (a, c, d, f) The concentration of NADPH oxidase (NOX) and peroxynitrite was elevated significantly in NAFLD and $\mathrm{H}_{2} \mathrm{O}_{2}$ groups, respectively. (b, e) The concentration of nitric oxide was lower in the NAFLD and $\mathrm{H}_{2} \mathrm{O}_{2}$ groups.

amino acids (dityrosine, kynurenine, N-formylkynurenine, and tryptophan) and advanced oxidation protein products (AOPP), as well as beta-amyloid formation.

2.4.1. NAFLD Model. Incubation with PA resulted in significantly higher dityrosine (PHG: $+12.2 \%$; NAFLD: $+126.5 \% p$ $<0.0001$; NAFLD+PHG: $+47.8 \% p<0.01$, NAFLD+NAC: +9\%; NAFLD+ALA: +17.1\%), kyneurenine (PHG: $-25.3 \%$; NAFLD: $+29.8 \% p<0.05$; NAFLD+PHG: $+10.5 \%$, NAFLD +NAC: $\quad-7.6 \%$; NAFLD+ALA: $-40.9 \% \quad p<0.001), \quad \mathrm{N}-$ formylkynurenine (PHG: $-34.2 \% p<0.01$; NAFLD: $+60.8 \%$ $p<0.0001$; NAFLD+PHG: $+27.8 \% p<0.05$; NAFLD+NAC:
$+17.1 \%$, NAFLD+ALA: $+46.3 \% p<0.001$ ), AOPP (PHG: $-13.7 \%$; NAFLD: $+58.6 \% p<0.0001$; NAFLD+PHG: $+9.5 \%$; NAFLD+NAC: $+2.8 \%$, NAFLD+ALA: $+5.7 \%)$, and amyloid cross structure formation (PHG: $-4.3 \%$; NAFLD: $+65.8 \%$ $p<0.0001$; NAFLD+PHG: $+279.5 \%$; NAFLD+NAC: $+28.6 \%$, NAFLD+ALA: $+42.5 \% p<0.01)$. Additionally, formation of dityrosine and AOPP was significantly higher in the NAFLD group than in other groups. Tryptophan levels were found to be lower in the NAFLD-treated group (PHG: +7\%; NAFLD: $-29.4 \% p<0.05$; NAFLD+PHG: $-12.2 \%$; NAFLD+NAC: $-8.8 \%$, NAFLD+ALA: $-1 \%)$ (Figure 5). 
2.4.2. $\mathrm{H}_{2} \mathrm{O}_{2}$ Model. Levels of dityrosine (PHG: $+12.2 \% ; \mathrm{H}_{2} \mathrm{O}_{2}$ : $+133.2 \% p<0.0001 ; \mathrm{H}_{2} \mathrm{O}_{2}+\mathrm{PHG}:+42.4 \% ; \mathrm{H}_{2} \mathrm{O}_{2}+\mathrm{NAC}$ : $+4.7 \% ; \mathrm{H}_{2} \mathrm{O}_{2}+\mathrm{ALA}:+27.1 \%$ ) and AOPP (PHG: $-13.7 \%$; $\mathrm{H}_{2} \mathrm{O}_{2}:+95.4 \% p<0.0001 ; \mathrm{H}_{2} \mathrm{O}_{2}+$ PHG: $+45.8 \% p<0.01$; $\mathrm{H}_{2} \mathrm{O}_{2}+\mathrm{NAC}:+37 \% p<0.05 ; \mathrm{H}_{2} \mathrm{O}_{2}+$ ALA: $+43.6 \% p<0.01$ ) were substantially elevated in the group exposed to $\mathrm{H}_{2} \mathrm{O}_{2}$. By comparison, concentration of tryptophan in the same group was moderately but significantly lower compared to that in the control (PHG: $+7 \% ; \mathrm{H}_{2} \mathrm{O}_{2}:-31.4 \% p<0.05$; $\mathrm{H}_{2} \mathrm{O}_{2}+$ PHG: $-10.5 \% ; \mathrm{H}_{2} \mathrm{O}_{2}+\mathrm{NAC}:-11.6 \% ; \mathrm{H}_{2} \mathrm{O}_{2}+$ ALA: $-15.2 \%)$. $\mathrm{N}$-formylkynurenine level was decreased in the PHG group and significantly elevated in other examined groups (PHG: $-34.2 \% p<0.01 ; \mathrm{H}_{2} \mathrm{O}_{2}:+70.4 \% p<0.0001$; $\mathrm{H}_{2} \mathrm{O}_{2}+$ PHG: $+50.4 \% p<0.0001 ; \mathrm{H}_{2} \mathrm{O}_{2}+\mathrm{NAC}:+37.9 \% p<$ $0.01 ; \mathrm{H}_{2} \mathrm{O}_{2}+$ ALA: $\left.+45.5 \% p<0.001\right)$. There was no effect on the amyloid cross structure observed (PHG: $-4.3 \%$; $\mathrm{H}_{2} \mathrm{O}_{2}:+11.8 \% ; \mathrm{H}_{2} \mathrm{O}_{2}+$ PHG: $+16.1 \% ; \mathrm{H}_{2} \mathrm{O}_{2}+\mathrm{NAC}:-15.2 \%$; $\mathrm{H}_{2} \mathrm{O}_{2}+$ ALA: $-4.7 \%$ ) (Figure 6).

2.5. Lipid and Carbonyl Damage. We assessed the oxidative damage to lipids by evaluating the concentration of malondialdehyde (MDA) and lipid hydroperoxides (LOOH). To assess carbonyl stress, we used advanced glycation end products (AGE).

2.5.1. NAFLD Model. It was revealed that $\mathrm{LOOH}$ and MDA levels were substantially increased in the group exposed to PA (PHG: $-17.6 \%$; NAFLD: $+66.8 \% p<0.0001$; NAFLD +PHG: $-6.7 \%, \quad$ NAFLD+NAC: $-11.3 \%, \quad$ NAFLD+ALA: $-3.8 \%$ ) (PHG: $+6.5 \%$; NAFLD: $+64.7 \% p<0.0001$; NAFLD + PHG: $+15.7 \%$, NAFLD+NAC: $+5.7 \%$, NAFLD +ALA:+1.7\%) whereas AGE levels rose in NAFLD, NAFLD + PHG, NAFLD+NAC, and NAFLD+ALA groups (PHG: $-1.2 \%$; NAFLD: $+144.8 \% \quad p<0.0001$; NAFLD+PHG: $+71.7 \% \quad p<0.0001 ; \quad$ NAFLD+NAC: $\quad+55.3 \% \quad p<0.01$; NAFLD+ALA: $+67.2 \% p<0.001)$ as compared to the control (Figure 7).

2.5.2. $\mathrm{H}_{2} \mathrm{O}_{2}$ Model. In comparison to the control, $\mathrm{LOOH}$ and MDA levels were increased significantly in the group treated with $\mathrm{H}_{2} \mathrm{O}_{2}, \mathrm{H}_{2} \mathrm{O}_{2}+\mathrm{PHG}, \mathrm{H}_{2} \mathrm{O}_{2}+\mathrm{NAC}$, and $\mathrm{H}_{2} \mathrm{O}_{2}+\mathrm{ALA}$ (PHG: $-17.6 \% ; \mathrm{H}_{2} \mathrm{O}_{2}$ : +123.2\% $p<0.0001 ; \mathrm{H}_{2} \mathrm{O}_{2}+$ PHG: $+51.4 \% \quad p<0.01 ; \quad \mathrm{H}_{2} \mathrm{O}_{2}+\mathrm{NAC}: \quad+39.7 \% \quad p<0.05 ; \quad \mathrm{H}_{2} \mathrm{O}_{2}$ +ALA: $+56.2 \% p<0.01$ ) (PHG: $+6.5 \% ; \mathrm{H}_{2} \mathrm{O}_{2}:+122.8 \% p<$ $0.0001 ; \mathrm{H}_{2} \mathrm{O}_{2}+$ PHG: $+71 \% p<0.0001 ; \mathrm{H}_{2} \mathrm{O}_{2}+\mathrm{NAC}:+72.5 \%$ $p<0.0001 ; \mathrm{H}_{2} \mathrm{O}_{2}+$ ALA: $+69.9 \% p<0.001$ ) while significant growth in AGE levels was registered in $\mathrm{H}_{2} \mathrm{O}_{2}, \mathrm{H}_{2} \mathrm{O}_{2}+\mathrm{NAC}$, and $\mathrm{H}_{2} \mathrm{O}_{2}+$ ALA sets (PHG: $-1.2 \% ; \mathrm{H}_{2} \mathrm{O}_{2}:+199.1 \% p<$ $0.0001 ; \mathrm{H}_{2} \mathrm{O}_{2}+$ PHG: $+39.8 \% ; \mathrm{H}_{2} \mathrm{O}_{2}+\mathrm{NAC}:+71.7 \% p<0.05$; $\mathrm{H}_{2} \mathrm{O}_{2}+$ ALA: $+79.5 \% p<0.01$ ) (Figure 7).

2.6. Inflammation, Hypoxia, and Apoptosis. The expression of proteins involved in the inflammatory and apoptotic pathways was assessed only in the main experimental and NAFLD model. PHG, both alone and combined with PA, decreased significantly the HIF- $1 \alpha$ level compared to the control (PHG: $-57.8 \%, \mathrm{PA}+\mathrm{PHG}$ : $-28.3 \%$; $p<0.05$ ). What is more, we found that PA incubation increased NF- $\kappa$ B expression (PA: $+80 \% ; p<0.05$ ), which was normalised when PHG was added to the steatotic medium (PA+PHG: $-100.4 \%$; $p$
$<0.01$ ). PHG also selectively inhibited COX-1 (PHG: $-66.8 \% ; p<0.01)$ but did not significantly affect COX-2 (Figure 8). Furthermore, both PHG and PA increased TNF $\alpha$ levels in the incubation media (PA: $+9.4 \%$; PHG: $+12.8 \%$, PA + PHG: $+24.2 \% ; p<0.05)$. PHG significantly lowered PA induced elevation of Il- $1 \beta$ (PA: $+32.4 \%, p<0.0001$; PA + PHG: $+10.3 \%, p<0.001)$ and Il-6 (PA: $+23.2 \%, p<0.01$; PA+PHG: $+5.2 \%, p<0.05$ ) (Figure 9). It was revealed that PA activated Caspase 9 (PA: $+59.4 \%$; $p<0.01$ ) and Caspase 3 (PA: $+83.7 \%$; $p<0.0001$ ), which was normalised when PHG was added to the incubation media (PA+PHG: $-25.5 \%$; $p<0.05$ ) (Figure 8).

\section{Discussion}

This is the first study to assess the effect of PHG on redox homeostasis and oxidative/nitrosative damage in HepG2 cells treated with palmitic acid and hydrogen peroxide. We have shown that PHG strengthens the enzymatic and nonenzymatic antioxidant barrier and prevents oxidative/nitrosative stress comparable to other commonly used antioxidants. Additionally, PHG reduces inflammation and apoptosis in the NAFLD model (Figure 10).

NAFLD is one of the most underestimated diseases in the XXI century. Despite its severe aftermaths, such as T2DM, obesity, liver cirrhosis, and HCC, commonly occurring in the developed countries, it still does not have an acknowledged and efficient treatment method [26, 27]. What is more, NAFLD, as a root cause of liver cirrhosis and HCC, became one of the main reasons for liver transplantation in countries with western dietary pattern [28]. NAFLD's clinical significance in creating its long-term effects may be explained by the "two-hit" theory [29]. Following this theory, the first hit is related to excessive lipid accumulation in the liver, mostly due to an unbalanced diet and overnutrition, resulting in hepatocyte steatosis. The second hit is represented by the lipotoxicity of accumulated lipids, leading to increased oxidative stress, impaired metabolic function, inflammatory process, and NASH development [30]. Indeed, the critical role of redox imbalance in the pathogenesis of fibrosis and steatosis in NAFLD patients has recently been highlighted [31]. Interestingly, there is a significant overproduction of hepatic hydrogen peroxide and progressive depletion of glutathione reserves in NAFLD patients, leading to enhanced protein and lipid oxidative injury. That is why finding new approaches to fight NAFLD and introducing them into clinical practice seems to be crucial to prevent the development of liver steatosis aftermath. So far, numerous compounds, such as resveratrol, quercetin, enterolactone, vitamin E, ALA, or NAC, have been tested for that purpose [27, 3236]. However, despite promising results in vitro, most of the polyphenols have very poor bioavailability in humans, and some have numerous side effects that limit their clinical usability. Thus, in our study, we focused on PHG, a phenolic antioxidant characterized by excellent pharmacokinetics, which may be found in seaweeds such as Ecklonia cava or Cystoseira discors $[22,37,38]$. PHG was discovered in 1855 , yet it was not used in applications other than as an antispasmodic drug. It has a similar structure to resveratrol, and as we 


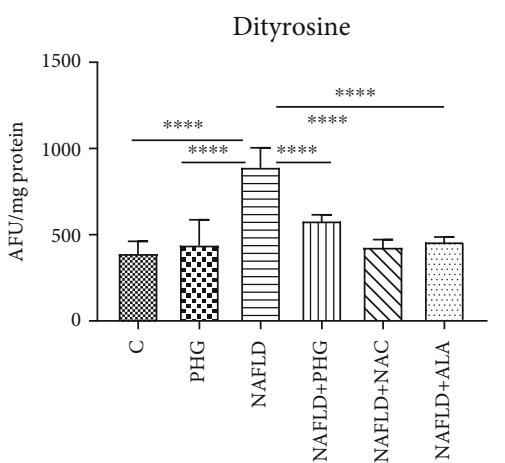

(a)

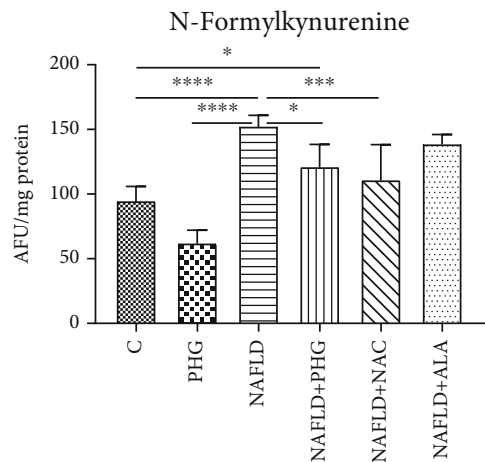

(c)

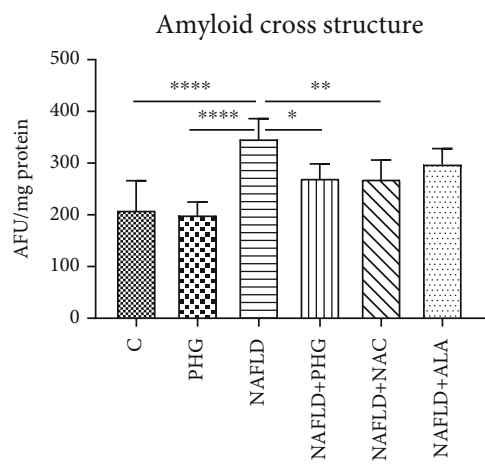

(e)

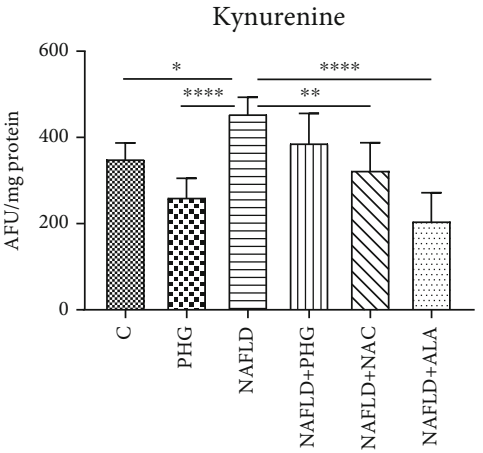

(b)

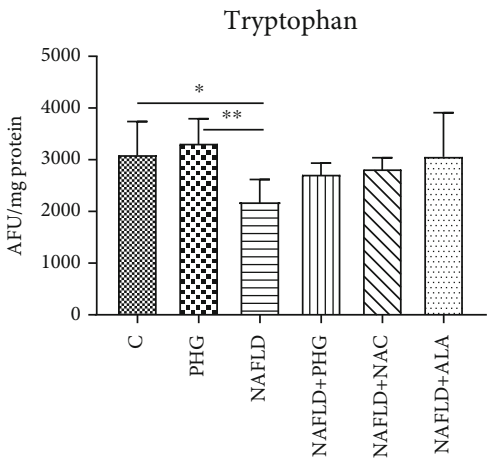

(d)

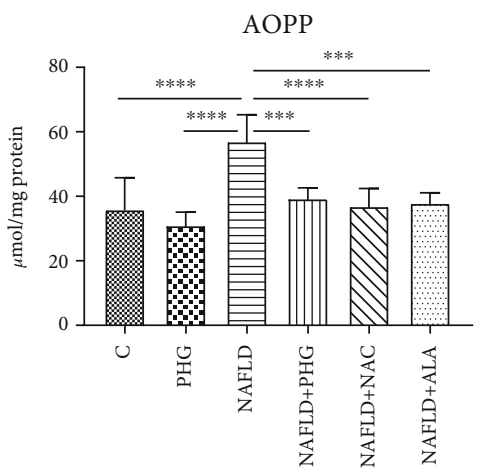

(f)

FIGURE 5: Protein glycooxidation damage in the NAFLD model. The concentration of the protein damage products in HepG2 cell cultures incubated with phloroglucinol (PHG), palmitate (NAFLD), both PHG and NAFLD, and palmitate with other known antioxidants: Nacetylcysteine (NAFLD+NAC) and alpha-lipoic acid (NAFLD+ALA). (a, b, e, f) The content of dityrosine, kynurenine, advanced oxidation protein products (AOPP), and the amyloid cross structure was increased significantly only in the NAFLD group. (c) The concentration of N-formylkynurenine was significantly elevated in the NAFLD group with a slight increase in the NAFLD+PHG group compared to the control (d). Concentration of tryptophan was decreased after incubation in NAFLD conditions compared with those of the control and PHG groups.

show herewith, similar to it, PHG decreases liver steatosis, strengthens antioxidative barriers, and reduces inflammation. In the following study, we assessed properties of PHG both in NAFLD and in hydrogen peroxide model and compared its effects with routinely used antioxidants-NAC and ALA.

In NAFLD, the excesses of saturated fatty acids, especially palmitic acid, accumulated in the liver inducing oxidative stress due to impaired mitochondrial $\beta$-oxidation and the generation of lipotoxic intermediates such as ceramides, diacylglycerols, and lysophosphatidylcholine [30]. A signifi- cant source of oxidative stress at NAFLD is the overproduction of hydrogen peroxide, so we decided on a second experimental model in our study. 50\% growth inhibition $\left(\mathrm{GI}_{50}\right)$ of $\mathrm{H}_{2} \mathrm{O}_{2}$ equal to around $10 \mathrm{mM}$ was obtained in the MTT test. PHG was nontoxic to HepG2 cells in concentrations below $200 \mu \mathrm{M}$. It reduced visible lipid accumulation and decreased the active form of Caspase 3 in the palmateinduced steatosis model (Figure 1). Since overproduction of ROS and reduced antioxidant defense are among the effects of excessive lipid accumulation in the liver, we analyzed the cellular content of enzymatic and nonenzymatic antioxidants 


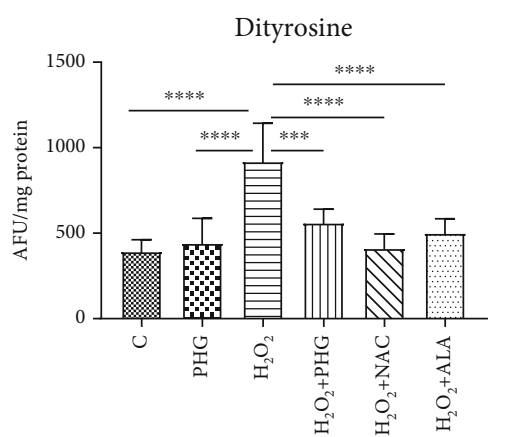

(a)

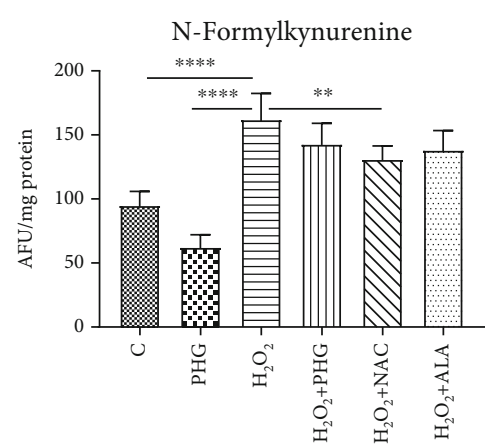

(c)

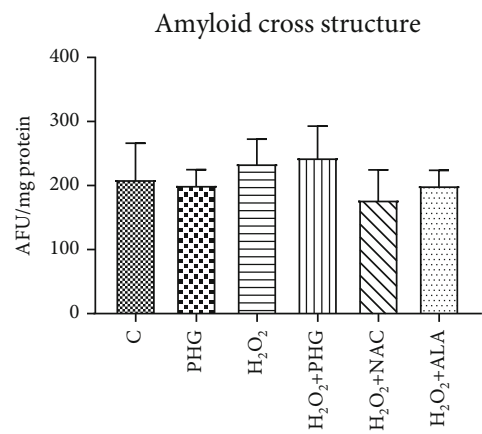

(e)

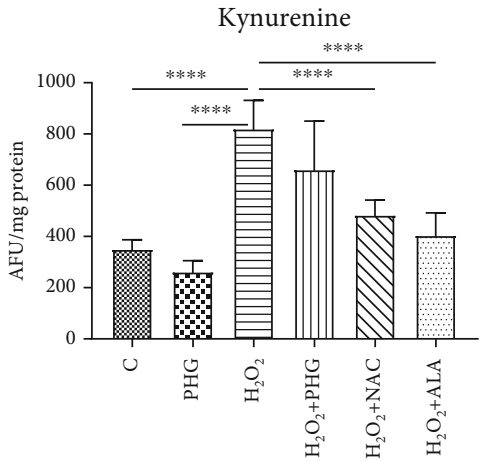

(b)

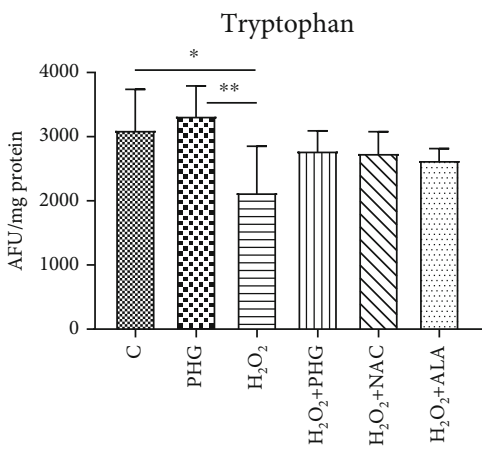

(d)

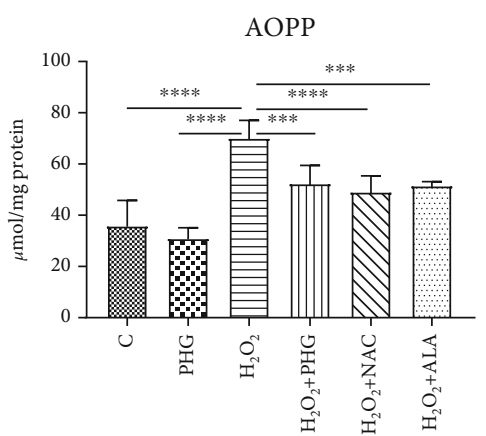

(f)

Figure 6: Protein glycooxidation damage in the $\mathrm{H}_{2} \mathrm{O}_{2}$ model. The concentration of the protein damage products in HepG2 cell cultures incubated with phloroglucinol (PHG), hydrogen peroxide $\left(\mathrm{H}_{2} \mathrm{O}_{2}\right)$, both PHG and $\mathrm{H}_{2} \mathrm{O}_{2}$, and hydrogen peroxide with other known antioxidants: N-acetylcysteine $\left(\mathrm{H}_{2} \mathrm{O}_{2}+\mathrm{NAC}\right)$ alpha-lipoic acid $\left(\mathrm{H}_{2} \mathrm{O}_{2}+\mathrm{ALA}\right)$. (a-c, f) The content of dityrosine, kynurenine, Nformylkynurenine, and advanced oxidation protein products (AOPP) was raised in the $\mathrm{H}_{2} \mathrm{O}_{2}$ group only. (d) The content of tryptophan was significantly lower in the $\mathrm{H}_{2} \mathrm{O}_{2}$ group compared to the control and PHG. (e) No differences in the concentration of amyloid cross structure between groups were observed.

$[13,14]$. The exposure to both palmitate and $\mathrm{H}_{2} \mathrm{O}_{2}$ resulted in a decreased level of reduced glutathione (GSH), which is the major nonenzymatic antioxidant in the liver [14, 39]. The addition of antioxidants is accompanied by an increase of enzymes involved in the restoration of a reduced form of glutathione (GR, GSH-Px) and other enzymatic antioxidants such as SOD and CAT. These effects were observed in both experimental models with a slight predominance of the NAFLD model. Interestingly, within all analyzed compounds (NAC, ALA, and PHG), PHG had the most decisive influence on the enzymatic antioxidant barrier (Figures 2 and 3 ). Only the increase in GSH level and redox ratio was significantly lower compared to NAC and ALA. However, this should not come as a surprise because the latter are direct precursors to glutathione biosynthesis. This is particularly important because NAC and ALA have a proven therapeutic effect in NAFLD therapy. However, their limited use is due to the need to administer very high doses (up to $0.5 \mathrm{~g}$ per $\mathrm{kg}$ of body weight), causing numerous side effects $[35,40]$. Although it is difficult to predict the side effects of PHG without human studies, we do not know any of the harmful actions of this compound. As far as enzymatic and nonenzymatic antioxidants are concerned, our observations are consistent with Quéguineur et al., who analyzed the dosedependent effects of PHG on tert-butyl hydroperoxideinduced oxidative stress [41]. Despite the differences in used concentrations and experimental models, this seems to confirm the beneficial role of PHG in strengthening the liver 


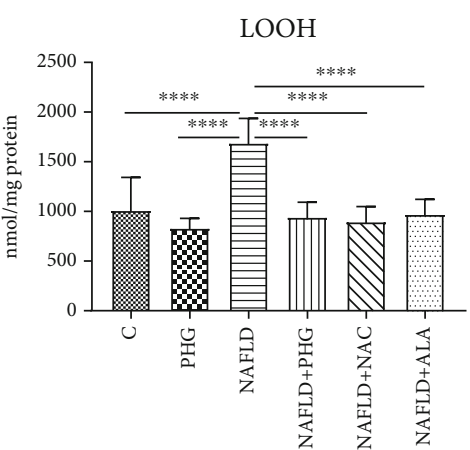

(a)

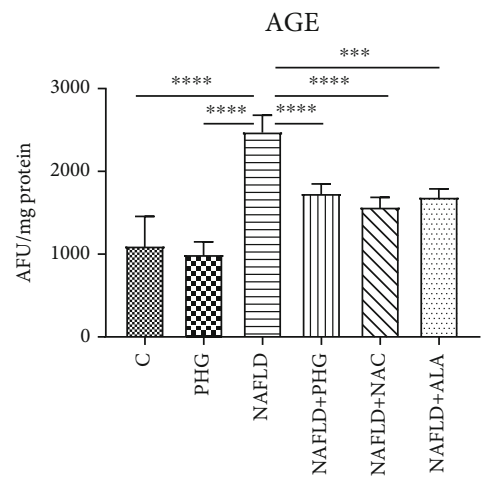

(c)

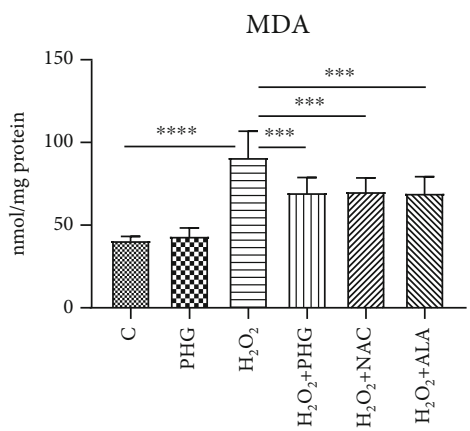

(e)

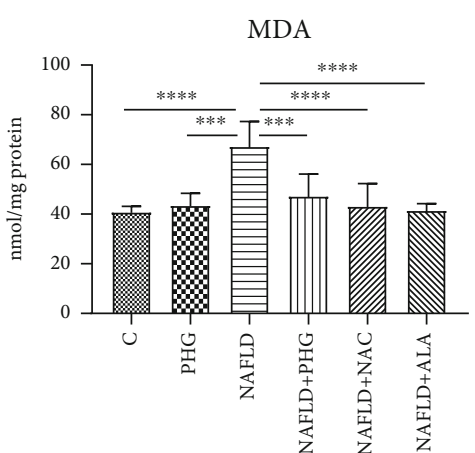

(b)

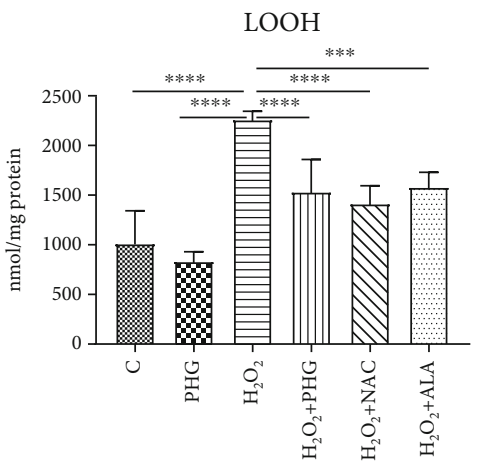

(d)

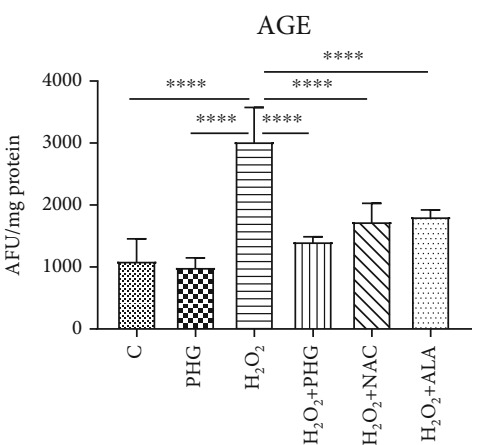

(f)

FIgURE 7: Lipid and carbonyl damage. The concentration of the lipid and carbonyl damage products in HepG2 cell cultures incubated with (first row) phloroglucinol (PHG), palmitate (NAFLD), both PHG and NAFLD, and palmitate with other known antioxidants: Nacetylcysteine (NAFLD+NAC) and alpha-lipoic acid (NAFLD+ALA); (second row) phloroglucinol (PHG), hydrogen peroxide $\left(\mathrm{H}_{2} \mathrm{O}_{2}\right)$, both PHG and $\mathrm{H}_{2} \mathrm{O}_{2}$, and hydrogen peroxide with other known antioxidants: $\mathrm{N}$-acetylcysteine $\left(\mathrm{H}_{2} \mathrm{O}_{2}+\mathrm{NAC}\right)$ alpha-lipoic acid $\left(\mathrm{H}_{2} \mathrm{O}_{2}\right.$ +ALA). (a-f) Concentration of total hydroperoxides (LOOH), malondialdehyde (MDA), and advanced glycation end products (AGE) was markedly elevated only in groups incubated in NAFLD and $\mathrm{H}_{2} \mathrm{O}_{2}$ conditions alone.

antioxidant defense. On the other hand, PHG, in contrast to myricetin and pyrogallol, failed to increase antioxidant defense in yeast Saccharomyces cerevisiae what might suggest an animal-specific effect of PHG [42]. Although we did not assess the rate of hydrogen peroxide production, probably, the observed increase in GSH-Px and CAT activity does not result from the adaptive reaction to the increased formation of $\mathrm{H}_{2} \mathrm{O}_{2}$ by phloroglucinol. Previous studies showed that PHG elevates CAT activity and its protein expression, while CAT inhibitor abolished the protective effect of PHG from $\mathrm{H}_{2} \mathrm{O}_{2}$-induced cellular damage [43].

In animals, the primary source of ROS generation in physiological conditions is $\beta$-oxidation. However, in
NAFLD, lipotoxic intermediates created by the incomplete oxidation of acyl-carnitine may also constitute an additional source of ROS [14]. Liver steatosis and exposure to $\mathrm{H}_{2} \mathrm{O}_{2}$ may also activate membrane NADPH oxidase (NOX), which catalase the process of superoxide anion formation [44]. Under these conditions, NO is also synthesized by inducible NO synthase (iNOS). iNOS is present in the liver and may be upregulated due to steatosis, cirrhosis, and liver cholestasis $[45,46]$. Interestingly, the interaction of superoxide anions with NO results in highly reactive peroxynitrite, which might explain lowered NO concentration in experimental models. Indeed, peroxynitrite is one of the strongest prooxidizing factors in living organisms [47, 48]. Peroxynitrite and its 


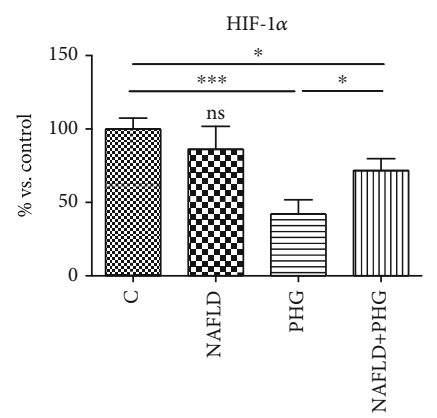

(a)

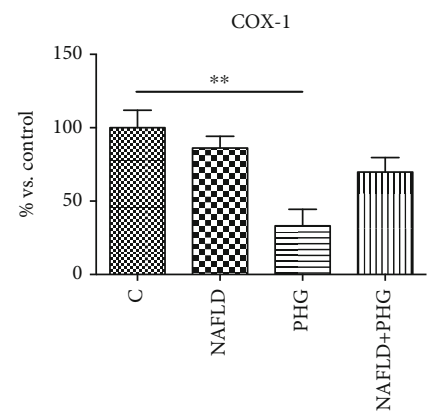

(c)

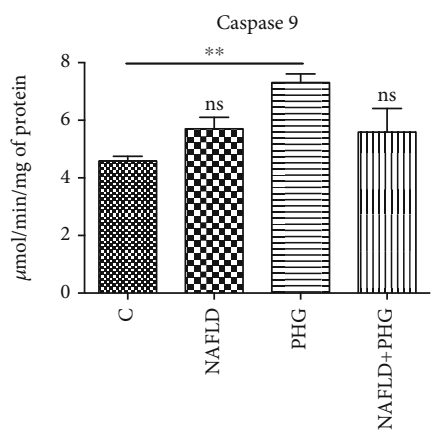

(e)

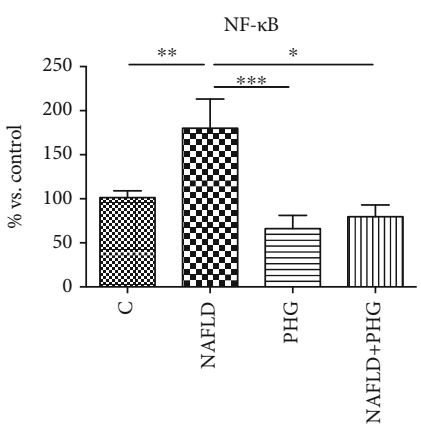

(b)

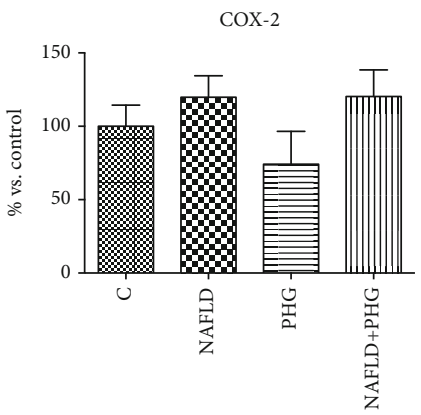

(d)

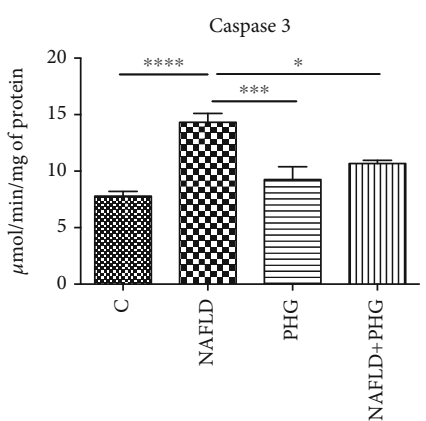

(f)

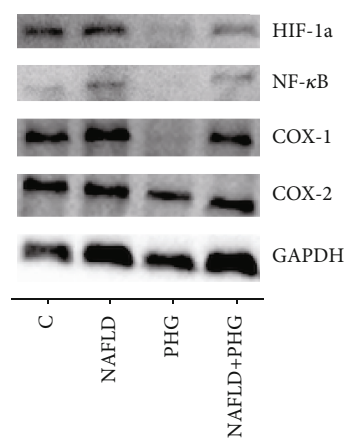

(g)

Figure 8: Inflammation, hypoxia, and apoptosis. PHG significantly reduced HIF- $1 \alpha$ level (a) and PA induced NF- $\kappa$ B activation (b) in HepG2 cells. Furthermore, it selectively inhibited COX-1 (c) with no effect on COX-2 (d). PHG stimulated Caspase 9 (e) and inhibited steatosisinduced activation of Caspase 3, showing its antiapoptotic properties (f). The expression of proteins involved in the inflammatory process assessed in Western blot (g). The band intensity was calculated as a \% of control.

derivatives react both with amino acids (including tyrosine, cysteine, and tryptophan), lipids, and several antioxidants. Peroxynitrite causes the formation of carbonyl groups, dimerization, nitration, and nitrosylation of amino acids and thiol compounds [48-50]. It was shown that nitrosative stress plays a critical role in various pathological conditions such as cardiovascular diseases, liver cirrhosis, diabetes, or cancer $[22,51]$. In our experiment, we observed that all 


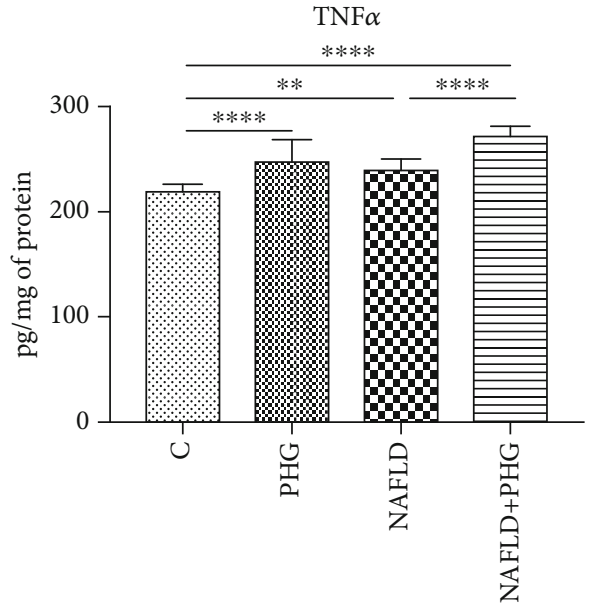

(a)

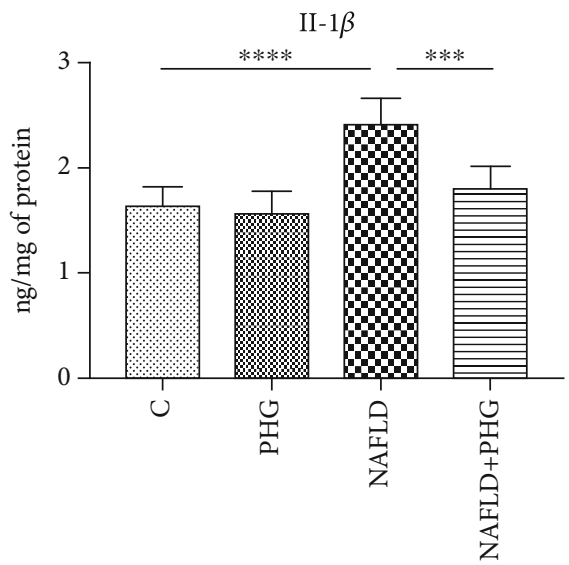

(b)

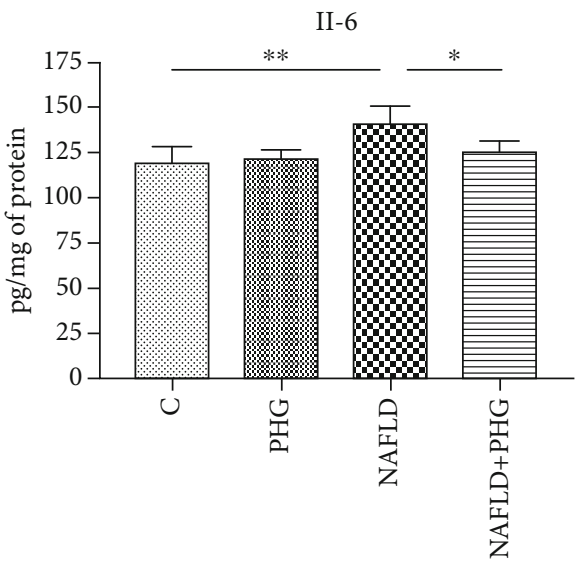

(c)

FIGURE 9: Proinflammatory cytokines. The TNF $\alpha$ concentration in the incubation media was slightly elevated in all the experimental groups when comparing to the control (a). Cell steatosis significantly increased the media level of main proinflammatory interleukins: Il- $1 \beta$ and Il-6 which were normalised when PHG was added $(b, c)$.

analyzed antioxidants presented a similar ability to diminish nitrosative stress both in the NAFLD and $\mathrm{H}_{2} \mathrm{O}_{2}$ models (Figure 4).

Once we established the antioxidant status and the level of the primary oxidative stress sources, we wanted to verify if PHG could prevent the formation of oxidative damage products. One of the most commonly used markers of overall protein damage is AOPP, which is created in interaction with chlorinated oxidants [52]. AOPP, similarly to MDA, CAT, and serum lipids, have been used recently to create a multimarker test aimed at improving the early identification of NAFLD and prediabetic patients [53]. As depicted in Figures 5 and 6 , all analyzed compounds were efficient in decreasing AOPP. The increase of AOPP in experimental models can correspond with the lowered cellular GSH level (Figures 2 and 3) which suggests that protein oxidation in the liver is a result of a diminished antioxidant barrier [54]. Other commonly used markers of ROS generated protein damage are oxidized forms of tryptophan: Nformylkynurenine and kynurenine. N-formylkynurenine results from posttranslational oxidation of tryptophan, which may be further converted into kynurenine [55]. ALA was the most potent among tested antioxidants in diminishing kynurenine, while PHG and NAC were more efficient in lowering the level of $\mathrm{N}$-formylkynurenine (Figures 5 and 6). Furthermore, we evaluated the level of dityrosine; a ROSmodified amino acid responsible for amyloid cross-linking, and the generation of $\mathrm{A} \beta$ plaques [56]. Both exposures to palmitate and $\mathrm{H}_{2} \mathrm{O}_{2}$ resulted in a significant increase of dityrosine, which was normalised in the presence of antioxidants. Interestingly, elevated amyloid cross structure content was observed only in the NAFLD model and partly decreased due to PHG and NAC action (Figures 5 and 6). Finally, we assessed the lipid and carbonyl damage products. Similar to proteins, both NAFLD and $\mathrm{H}_{2} \mathrm{O}_{2}$ caused a steep increase of oxidized forms of lipids such as MDA or LOOH and the elevation of glycation end products that were efficiently lowered when PHG, NAC, or ALA were added to the incubation media (Figure 7). Reducing the oxidation/nitrosylation of liver proteins and lipids may slow down the progression of NAFLD. It is well known that the increase in protein and lipid glycation is responsible for the development of ischemia and hepatic fibrosis, and thus, the progression of NAFL to NASH [14-57]. The accumulation of AGE and AOPP in 


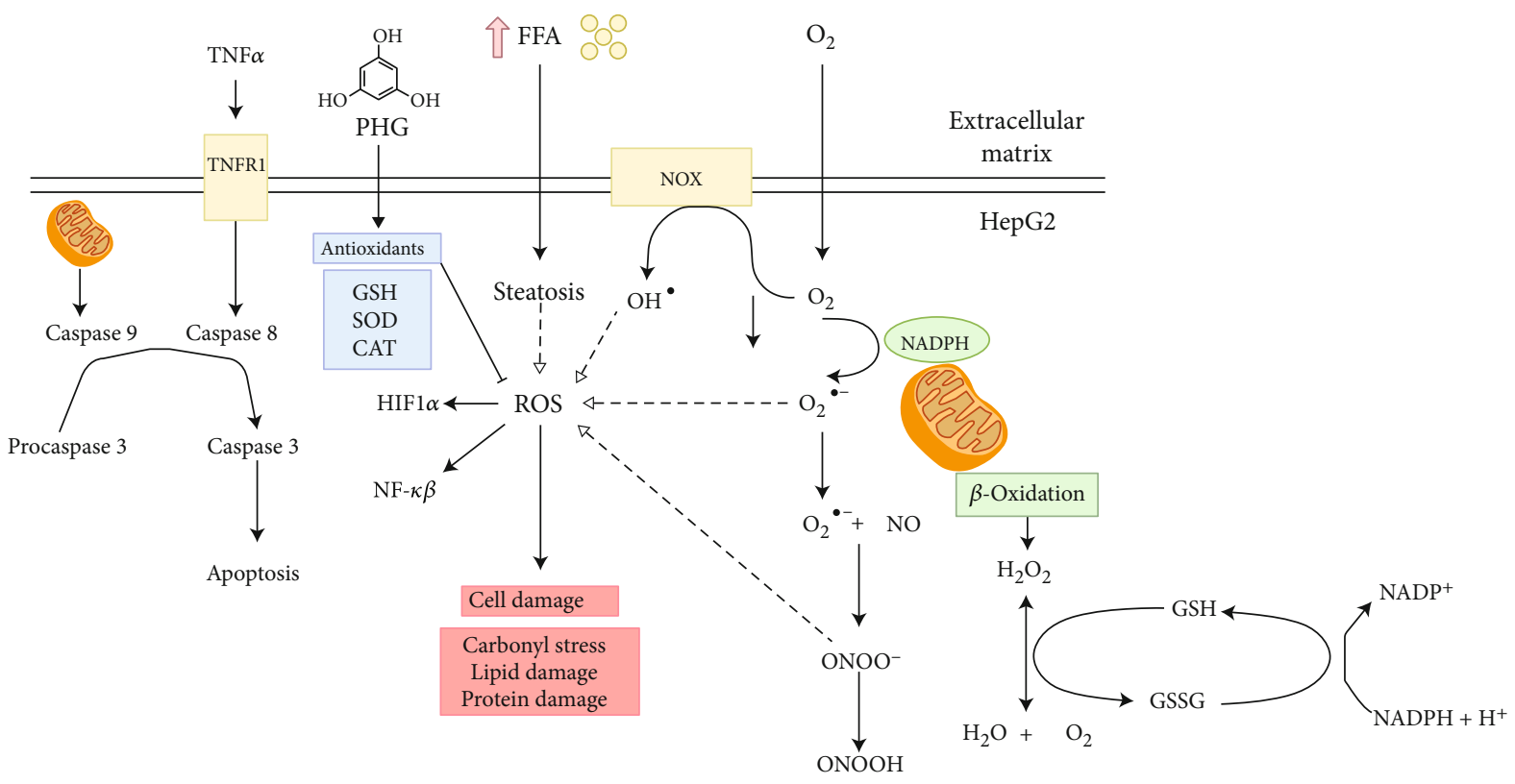

FIGURE 10: The potential mechanism underlying oxidative/nitrosative stress in nonalcoholic fatty liver disease (NAFLD).

the liver not only will increase the production of ROS (through NOX induction in the positive feedback mechanism) but also increases the expression of Fas ligand (protein from tumor necrosis factor (TNF) family) and activates the $\mathrm{NF}-\kappa \mathrm{B}$ transcription factor (nuclear factor kappa-lightchain-enhancer of activated B cells), which stimulates neutrophil chemotaxis in the hepatocytes $[31,58]$ In our study, PHG, by strengthening the antioxidant barrier, not only reduces ROS production and oxidative/glycooxidation damage to proteins and lipids but also prevents excessive nitrosylation of the cell.

To assess the influence of PHG on inflammation and cell survival, we analyzed the expression of key proteins involved in apoptosis. As depicted in Figures 1 and 8, PHG revealed antiapoptotic properties by inhibiting the caspase cascade in the steatotic liver. The drop in Caspase 3 activity corresponded to lowered HIF- $1 \alpha$ which might explain increased HepG2 survival. HIF- $1 \alpha$ upregulates FOXO3, which is responsible for promoting Bax over Bcl-2 signaling [59]. In contrast to antiapoptotic Bcl-2, Bax opens voltagedependent anion channels and creates pores in the mitochondrial outer membrane that initiate apoptosis [60]. Another interesting observation was a PHG-induced downregulation of NF- $\kappa \mathrm{B}$, a key regulator of proinflammatory signaling (Figure 8). As a result of its activation, NF- $\kappa$ B raises the TNF $\alpha$ level and stimulates iNOS, and COX-2 leads to proinflammatory prostaglandins and NO [61]. Despite the lack of statistically significant changes in COX-2, PHG inhibited COX-1 which may suggest its selectivity towards this isozyme (Figure 8). Nevertheless, the increased NO synthesis and inflammation results in oxidative/nitrosative stress and the creation of ROS and RNS, which weakens the antioxidative defense, and results in elevated protein accumulation, lipid, and carbonyl damage products. Finally, NF- $\kappa \mathrm{B}$ may also induce HIF- $1 \alpha$ expression as a downstream effect of the PI3K-Akt-NF- $\kappa$ B signaling pathway [62].
Our study confirms previous reports on the antioxidant properties of PHG. Indeed, it was shown that PHG protected human $\mathrm{HaCaT}$ keratinocytes against ultraviolet B- (UVB-) induced oxidative stress by scavenging intracellular ROS production [63]. PHG also decreased serum glucose level and formation of AGE in streptozotocin-induced diabetic rats [64]. Reduced oxidative stress under the influence of PHG was also noted in endothelial, neuronal, retinal, and neoplastic cells, which indicates the possibility of PHG as a promising therapeutic agent in several diseases [65-68]. A good pharmacokinetic profile and few PHG side effects are the additional advantages of this compound. However, as the mechanism of the antioxidant/antiglycation effect of PHG is not yet exactly known, further research is needed.

Nevertheless, our manuscript also has some limitations. We have evaluated the effect of PHG only on HepG2 cells, so further studies on other cell lines are necessary. Furthermore, we have only assessed selected oxidative/nitrosative stress biomarkers, so we cannot fully characterize the effect of PHG on NAFLD redox homeostasis. The next step is also to evaluate the therapeutic effect of PHG on the animal model and choose the dose characterizing the maximum therapeutic effect.

\section{Conclusions}

To sum up, our study showed that NAFLD and hydrogen peroxide models are comparable and suitable for assessing the oxidative/nitrosative stress in the liver. Although our study does not fully explain the PHG action's mechanism, this compound may be considered a new nutraceutical in counteracting NAFLD and preventing its severe molecular and clinical aftermath. Its effectiveness is comparable with other renowned antioxidants $\alpha$-lipoic acid and $\mathrm{N}$-acetylcysteine, which brings a promising perspective for the therapeutical application of phloroglucinol. The potential mechanism 
underlying oxidative/nitrosative stress in NAFLD are depicted on Figure 10.

\section{Materials and Methods}

5.1. Cell Culture. The study was conducted on HepG2 cells obtained from ATCC (American Type Culture Collection). The cells were incubated in DMEM (Dulbecco's modified Eagle's medium) enriched with $10 \%$ fetal bovine serum (FBS) and $1 \%$ penicillin/streptomycin for five days at $37^{\circ} \mathrm{C}$ in a humidified atmosphere containing $5 \%$ of $\mathrm{CO}_{2}$ until they will reach a confluence of $70 \%$. The media were changed every $48 \mathrm{~h}$ preceded by rinsing in PBS. Subsequently, cells were transferred to 6-well plates and cultured in the growth medium until they achieved $90 \%$ of confluence. Then experimental incubations were conducted. Subsequently, cells were scrubbed in ice-cold RIPA buffer containing protease and phosphatase inhibitors (Roche Diagnostics GmbH, Germany) and ultrasonicated (Hielscher UP50H, Germany).

5.2. Experimental Models. To induce steatosis, cells were serum-starved for five h in a medium deprived of glucose and then incubated for $16 \mathrm{~h}$ in media containing either $0.75 \mathrm{mM}$ palmitate alone or both PA and experimental compounds: $100 \mu \mathrm{M}$ PHG or $100 \mu \mathrm{M}$ ALA or $10 \mu \mathrm{M}$ NAC. Sodium palmitate was dissolved in absolute ethanol and heated to $70^{\circ} \mathrm{C}$ before conjugation with $10 \%$ fatty acid-free bovine serum albumin (BSA). Subsequently, the palmitic acid solution was added to serum-free DMEM supplemented with $10 \mathrm{mM}$ Hepes, similarly to previously described methods $[26,27,69]$. The $\mathrm{H}_{2} \mathrm{O}_{2}$ model was prepared simply by adding an appropriate amount of hydrogen peroxide to the standard growth medium. Sigma-Aldrich, Poland, provided all the compounds.

5.3. Redox Homeostasis. The performed analyses included determination of antioxidant enzymes [catalase (CAT), glutathione peroxidase (GSH-Px), glutathione reductase (GR), and superoxide dismutase (SOD)] and nonenzymatic antioxidants [GSH], determination of prooxidant enzymes (NADPH oxidase, NOX), determination of oxidative damage to proteins [advanced glycation end products (AGE) and advanced oxidation protein products (AOPP)] and lipids [malondialdehyde (MDA) and total lipid hydroperoxides $(\mathrm{LOOH})]$, and determination of protein glycooxidative products [dityrosine, kynurenine, $\mathrm{N}$-formylkynurenine, and tryptophan], as well as the determination of nitrosative stress products [nitric oxide (NO) and peroxynitrite]. The absorbance/fluorescence was analyzed using the Infinite M200 PRO Microplate Reader (Tecan, Männedorf, Switzerland). All results were standardized to $\mathrm{mg}$ of the total protein. In the analysis of redox homeostasis and oxidative damage products, we followed the methods of Maciejczyk et al. [70].

5.4. Enzymatic and Nonenzymatic Antioxidants. The activity of catalase (CAT, EC 1.11.1.6) was estimated using the colorimetric method by measuring hydrogen peroxide $\left(\mathrm{H}_{2} \mathrm{O}_{2}\right)$ decomposition at $240 \mathrm{~nm}[71,72]$. One unit of CAT activity was defined as the quantity of the enzyme catalyzing decomposition of $1 \mathrm{mmol} \mathrm{H}_{2} \mathrm{O}_{2}$ per $1 \mathrm{~min}$. The activity of glutathi- one peroxidase (GSH-Px, EC 1.11.1.9) was analyzed colorimetrically by measuring the NADPH oxidation at $340 \mathrm{~nm}$ [73]. One unit of GPx activity was defined as the quantity of enzyme catalyzing the oxidation of $1 \mathrm{mmol}$ NADPH per $1 \mathrm{~min}$. The activity of glutathione reductase (GR, EC 1.8.1.7) was analyzed colorimetrically by measuring the decrease in NADPH absorbance at $340 \mathrm{~nm}$ [74]. One unit of GR activity was defined as the amount of enzyme catalyzing the oxidation of $1 \mu \mathrm{mol}$ NADPH per $1 \mathrm{~min}$. The activity of superoxide dismutase (SOD, EC 1.15.1.1) was determined colorimetrically by measuring the inhibition of adrenaline oxidation at $480 \mathrm{~nm}$ [71]. One unit of SOD activity was defined as the quantity of enzyme inhibiting adrenaline oxidation by $50 \%$.

The level of total glutathione was measured based on an enzymatic reaction with 5, $5^{\prime}$-dithiobis-(2-nitrobenzoic acid) (DTNB), NADPH, and GR [75]. Oxidized glutathione (disulfide glutathione, GSSG) was determined similarly to the assay performed for total glutathione. However, prior to the determination, the samples had been thawed and neutralized to pH 6-7 using $1 \mathrm{M}$ chlorhydrol triethanolamine. Then, samples were incubated with 2-vinylpyridine. The level of reduced glutathione (GSH) was calculated from the difference between the level of total glutathione and disulfide glutathione [75]. Redox ratio was calculated using the formula $[\mathrm{GSH}]^{2} /[\mathrm{GSSG}][76]$.

5.5. ROS Production and Nitrosative Stress. NADPH oxidase activity (NOX, EC 1.6.3.1) was analyzed by the luminescence method using lucigenin as an electron acceptor $[77,78]$. One unit of NOX activity was defined as the quantity of enzyme required to release $1 \mathrm{nmol}$ of the superoxide anion per 1 min. The cells used for ROS production measurements were cultured in pyruvate and antibiotic-free media.

The concentration of nitric oxide (NO) was determined using the Griess method based on the reaction of nitrates with sulfanilamide and N-(1-naphthyl)-ethylenediamine dihydrochloride $[79,80]$. The absorbance was measured at $490 \mathrm{~nm}$. The concentration of peroxynitrite was estimated colorimetrically based on peroxynitrite-mediated nitration of phenol to nitrophenol [81]. The absorbance was measured at $320 \mathrm{~nm}$.

5.6. Protein Glycooxidation Products. The content of protein glycooxidation products (dityrosine, kynurenine, $\mathrm{N}$-formylkynurenine, and tryptophan) was estimated fluorimetrically by measuring fluorescence at $330 / 415 \mathrm{~nm}$ (dityrosine), 365/480 nm (kynurenine), 325/434 nm (N-formylkynurenine), and $95 / 340 \mathrm{~nm}$ (tryptophan). Immediately before the assay, cells were diluted in $0.1 \mathrm{M} \mathrm{H}_{2} \mathrm{SO}_{4}(1: 10, v / v)$. The results were normalised to fluorescence of $0.1 \mathrm{mg} / \mathrm{mL}$ quinine sulfate in $0.1 \mathrm{M} \mathrm{H} 2 \mathrm{SO} 4$ [54]. The concentration of advanced oxidation protein products (AOPP) was estimated colorimetrically by measuring the sample's iodide ion oxidizing capacity at $340 \mathrm{~nm}[10]$.

5.7. Lipid and Carbonyl Damage. The concentration of total hydroperoxides $(\mathrm{LOOH})$ was determined colorimetrically based on the reaction of $\mathrm{Fe}^{3+}$ (resulting from $\mathrm{Fe}^{2+}$ after its 
oxidation by $\mathrm{LOOH}$ ) with xylenol orange [82]. The absorbance of the resulting complex was measured at $560 \mathrm{~nm}$. Immediately before the assay, cells were diluted in $0.02 \mathrm{M}$ PBS, pH 7.4 $(1: 5, v: v)$ [71]. The concentration of malondialdehyde (MDA) was determined colorimetrically using the thiobarbituric acid reactive substances (TBARS) method. The absorbance was measured at $535 \mathrm{~nm}$, and 1,3,3,3-tetraethoxypropane was used as a standard [83]. The concentration of advanced glycation end products (AGE) was detected fluorimetrically by measuring AGE-specific fluorescence at 350/440 nm [84]. Immediately before the assay, cells were diluted in $0.02 \mathrm{M}$ phosphate-buffered saline (PBS) $\mathrm{pH} 7.4(1: 5, v: v)[83]$.

5.8. In Situ Immunofluorescence. Approximately $10^{6}$ cells were seeded on a 12 -well plate and grown overnight. The next day, cells were treated in the same manner as cells from the main part of the experiment. At the end of $16 \mathrm{~h}$, experimental incubation cells were rinsed with PBS and fixed in $3.7 \%$ paraformaldehyde for $15 \mathrm{~min}$, permeabilized with $0.1 \%$ Triton X-100 for $20 \mathrm{~min}$, and blocked with $2 \%$ FBS $1 \%$ BSA in PBS for $30 \mathrm{~min}$ at room temperature. Subsequently, cells were incubated with the primary monoclonal anticleaved Caspase 3 antibody (1:200, Abcam, UK) and then with the secondary goat-anti-rabbit antibody conjugated with Alexa Fluor 488 (1:1000, Abcam, UK) both for $1 \mathrm{~h}$ at room temperature. Finally, cell nuclei were counterstained with DAPI (1:5000, Sigma-Aldrich). The images were obtained with a fluorescent microscope (Leica DMi8, Germany).

5.9. Oil Red O Staining. Approximately $10^{6}$ cells were seeded on a 12 -well plate and grown overnight. The next day, cells were treated in the same manner as cells from the main part of the experiment. After experimental treatment, cells were fixed in $3.7 \%$ paraformaldehyde for $15 \mathrm{~min}$ and then stained with $0.5 \%$ Oil Red O solution. The images were obtained with a fluorescent microscope (Leica DMi8, Germany).

5.10. Western Blotting. Proteins of interest expression were analyzed using the standard Western blot technique. To standardize samples, total protein concentration was assessed using the bicinchoninic acid method (BCA) with BSA as a standard. Cell lysates were separated by $10 \%$ sodium dodecyl sulfate-polyacrylamide gel electrophoresis (SDS-PAGE) and transferred to nitrocellulose membranes. Subsequently, they were blocked with 5\% nonfat dry milk and immunoblotted with primary antibodies of interest and incubated with secondary antibodies labeled with horseradish peroxidase (HRP). The protein bands were quantified densitometrically using the ChemiDoc visualisation system (Bio-Rad, Poland). Equal protein loading was controlled by Ponceau S staining. All the proteins' expression was standardized to the GAPDH (Santa Cruz Biotechnology, USA) expression, and the control was set as $100 \%$.

5.11. ELISA. TNF $\alpha$, IL-1, and IL-6 concentrations were analyzed using a standard ELISA kit purchased from Abcam, UK (TNF $\alpha$ ) and EIAab, China (IL-1 and IL-6). All the procedures were made on cell culture media samples standardized to protein concentration following the manufacturer's instructions. The assay was done in triplicate, and the results were averaged.

5.12. Statistical Analysis. The results were expressed as mean \pm SD based on six independent repetitions. Statistical significance was tested with one-way analyses of variance (ANOVA) and Tukey HSD post hoc test using GraphPad Prism 7 (GraphPad Software Inc., La Jolla, CA, USA). Multiplicity adjusted $p$ value was also calculated. Results were considered statistically significant at $p \leq 0.05$.

\section{Data Availability}

The data used to support the findings of this study are available from the corresponding author upon request.

\section{Conflicts of Interest}

The authors declare no conflict of interest.

\section{Authors' Contributions}

K. D. and K. S. contributed equally to this work.

\section{Acknowledgments}

The project was financed within the framework of the National Science Centre, Poland (grant number: 2018/31/N/NZ7/03242) and Polish Ministry of Science and Higher Education program "Strategy of Excellence-the University of Research" in the years 2018-2019 (project no.: 0017/SDU/2018/18; the amount of funding PLN 690,000) and by grants from the Medical University of Bialystok, Poland (grant numbers: SUB/1/DN/20/003/4406, SUB/1/DN/20/002/1209, and SUB/1/DN/20/002/3330). Mateusz Maciejczyk, PhD, was supported by the Foundation for Polish Science (FNP).

\section{References}

[1] M. E. Rinella, "Nonalcoholic fatty liver disease: a systematic review," JAMA, vol. 313, no. 22, pp. 2263-2273, 2015.

[2] Z. M. Younossi, G. Marchesini, H. Pinto-Cortez, and S. Petta, "Epidemiology of nonalcoholic fatty liver disease and nonalcoholic steatohepatitis: implications for liver transplantation," Transplantation, vol. 103, no. 1, pp. 22-27, 2019.

[3] B. Gao and H. Tsukamoto, "Inflammation in alcoholic and nonalcoholic fatty liver disease: friend or foe?," Gastroenterology, vol. 150, no. 8, pp. 1704-1709, 2016.

[4] T. Jelenik, K. Kaul, G. Séquaris et al., "Mechanisms of insulin resistance in primary and secondary nonalcoholic fatty liver," Diabetes, vol. 66, no. 8, pp. 2241-2253, 2017.

[5] B. Fruci, S. Giuliano, A. Mazza, R. Malaguarnera, and A. Belfiore, "Nonalcoholic fatty liver: a possible new target for type 2 diabetes prevention and treatment," International Journal of Molecular Sciences, vol. 14, no. 11, pp. 2293322966, 2013.

[6] L. A. Adams, O. R. Waters, M. W. Knuiman, R. R. Elliott, and J. K. Olynyk, "NAFLD as a risk factor for the development of diabetes and the metabolic syndrome: an eleven-year follow- 
up study," The American Journal of Gastroenterology, vol. 104, no. 4, pp. 861-867, 2009.

[7] S. Wu, F. Wu, Y. Ding, J. Hou, J. Bi, and Z. Zhang, "Association of non-alcoholic fatty liver disease with major adverse cardiovascular events: a systematic review and meta-analysis," Scientific Reports, vol. 6, no. 1, article 33386, p. 1, 2016.

[8] C. Söderberg, P. Stål, J. Askling et al., "Decreased survival of subjects with elevated liver function tests during a 28-year follow-up," Hepatology, vol. 51, no. 2, pp. 595-602, 2010.

[9] C. R. Wong and J. K. Lim, "The association between nonalcoholic fatty liver disease and cardiovascular disease outcomes," Clinics in Liver Disease, vol. 12, no. 2, pp. 39-44, 2018.

[10] S. Spahis, E. Delvin, J. M. Borys, and E. Levy, "Oxidative stress as a critical factor in nonalcoholic fatty liver disease pathogenesis," Antioxidants and Redox Signaling, vol. 26, no. 10, pp. 519-541, 2017.

[11] F. Ucar, S. Sezer, S. Erdogan, S. Akyol, F. Armutcu, and O. Akyol, "The relationship between oxidative stress and nonalcoholic fatty liver disease: Its effects on the development of nonalcoholic steatohepatitis," Redox Report, vol. 18, no. 4, pp. 127-133, 2013.

[12] M. Maciejczyk, A. Skutnik-Radziszewska, I. Zieniewska et al., "Antioxidant defense, oxidative modification, and salivary gland function in an early phase of cerulein pancreatitis," Oxidative Medicine and Cellular Longevity, vol. 2019, Article ID 8403578, 14 pages, 2019.

[13] I. Grattagliano, O. de Bari, T. C. Bernardo, P. J. Oliveira, D. Q. $\mathrm{H}$. Wang, and P. Portincasa, "Role of mitochondria in nonalcoholic fatty liver disease-from origin to propagation," Clinical Biochemistry, vol. 45, no. 9, pp. 610-618, 2012.

[14] M. Masarone, V. Rosato, M. Dallio et al., "Role of oxidative stress in pathophysiology of nonalcoholic fatty liver disease," Oxidative Medicine and Cellular Longevity, vol. 2018, Article ID 9547613, 14 pages, 2018.

[15] E. Kathirvel, P. Chen, K. Morgan, S. W. French, and T. R. Morgan, "Oxidative stress and regulation of anti-oxidant enzymes in cytochrome P4502E1 transgenic mouse model of nonalcoholic fatty liver," Journal of Gastroenterology and Hepatology, vol. 25, no. 6, pp. 1136-1143, 2010.

[16] K. Tsunoda, Y. Kai, N. Kitano, K. Uchida, T. Kuchiki, and T. Nagamatsu, "Impact of physical activity on nonalcoholic steatohepatitis in people with nonalcoholic simple fatty liver: a prospective cohort study," Preventive Medicine, vol. 88, pp. 237-240, 2016.

[17] A. Cicero, A. Colletti, and S. Bellentani, "Nutraceutical approach to non-alcoholic fatty liver disease (NAFLD): the available clinical evidence," Nutrients, vol. 10, no. 9, article 1153, 2018.

[18] E. A. Klein, I. M. Thompson, C. M. Tangen et al., "Vitamin E and the risk of prostate cancer: the Selenium and Vitamin E Cancer Prevention Trial (SELECT)," December 2020, http:// www.jama.com.

[19] M. Schürks, R. J. Glynn, P. M. Rist, C. Tzourio, and T. Kurth, "Effects of vitamin E on stroke subtypes: meta-analysis of randomised controlled trials," BMJ, vol. 341, no. 7781, article 1033, 2010.

[20] R. Marchioli, G. Levantesi, A. Macchia et al., "Vitamin E increases the risk of developing heart failure after myocardial infarction: results from the GISSI-Prevenzione trial," Journal of Cardiovascular Medicine, vol. 7, no. 5, pp. 347350, 2006 .
[21] A. Annaházi, R. Róka, A. Rosztóczy, and T. Wittmann, "Role of antispasmodics in the treatment of irritable bowel syndrome," World Journal of Gastroenterology, vol. 20, no. 20, pp. 6031-6043, 2014.

[22] M. J. So and E. J. Cho, "Phloroglucinol attenuates free radicalinduced oxidative stress," Preventive Nutrition and Food Science, vol. 19, no. 3, pp. 129-135, 2014.

[23] Y. Q. He, W. T. Zhang, C. H. Shi, F. M. Wang, X. J. Tian, and L. L. Ma, "Phloroglucinol protects the urinary bladder via inhibition of oxidative stress and inflammation in a rat model of cyclophosphamide-induced interstitial cystitis," Chinese Medical Journal, vol. 128, no. 7, pp. 956-962, 2015.

[24] R.-K. Kim, Y. Suh, K.-. C. Yoo et al., "Phloroglucinol suppresses metastatic ability of breast cancer cells by inhibition of epithelial-mesenchymal cell transition," Cancer Science, vol. 106, no. 1, pp. 94-101, 2015.

[25] M. H. Kang, I. H. Kim, and T. J. Nam, "Phloroglucinol induces apoptosis via apoptotic signaling pathways in HT-29 colon cancer cells," Oncology Reports, vol. 32, no. 4, pp. 1341-1346, 2014.

[26] M. V. Machado and H. Cortez-Pinto, "Non-alcoholic fatty liver disease: what the clinician needs to know," World Journal of Gastroenterology, vol. 20, no. 36, pp. 12956-12980, 2014.

[27] T. Yan, N. Yan, P. Wang et al., "Herbal drug discovery for the treatment of nonalcoholic fatty liver disease," Acta Pharmaceutica Sinica B, vol. 10, no. 1, pp. 3-18, 2020.

[28] P. Angulo, "Nonalcoholic fatty liver disease and liver transplantation," Liver Transplantation, vol. 12, no. 4, pp. 523534, 2006.

[29] C. P. Day and O. F. W. James, "Hepatic steatosis: innocent bystander or guilty party?," Hepatology, vol. 27, no. 6, pp. 1463-1466, 1998.

[30] W. Peverill, L. Powell, and R. Skoien, "Evolving concepts in the pathogenesis of NASH: beyond steatosis and inflammation," International Journal of Molecular Sciences, vol. 15, no. 5, pp. 8591-8638, 2014.

[31] M. Świderska, M. Maciejczyk, A. Zalewska, J. Pogorzelska, R. Flisiak, and A. Chabowski, "Oxidative stress biomarkers in the serum and plasma of patients with non-alcoholic fatty liver disease (NAFLD). Can plasma AGE be a marker of NAFLD? Oxidative stress biomarkers in NAFLD patients," Free Radical Research, vol. 53, no. 8, pp. 841-850, 2019.

[32] K. Drygalski, K. Berk, T. Charytoniuk et al., "Does the enterolactone (ENL) affect fatty acid transporters and lipid metabolism in liver?," Nutrition \& Metabolism, vol. 14, no. 1, p. 69, 2017.

[33] K. Berk, K. Drygalski, E. Harasim-Symbor et al., "The effect of enterolactone on liver lipid precursors of inflammation," Life Sciences, vol. 221, pp. 341-347, 2019.

[34] M. N. Stanković, D. Mladenović, M. Ninković et al., "The effects of $\alpha$-lipoic acid on liver oxidative stress and free fatty acid composition in methionine-choline deficient dietinduced NAFLD," Journal of Medicinal Food, vol. 17, no. 2, pp. 254-261, 2014.

[35] M. Khoshbaten, A. Aliasgarzadeh, K. Masnadi et al., "N-acetylcysteine improves liver function in patients with nonalcoholic fatty liver disease," Hepatitis Monthly, vol. 10, no. 1, pp. 12-16, 2010.

[36] T. Charytoniuk, E. Harasim-Symbor, A. Polak et al., "Influence of resveratrol on sphingolipid metabolism in hepatocellular carcinoma cells in lipid overload state," Anti-Cancer Agents in Medicinal Chemistry, vol. 19, no. 1, pp. 121-129, 2019. 
[37] G. Dollo, F. Chevanne, P. Le Corre, C. Chemtob, and R. Le Verge, "Biodisponibilite du phloroglucinol chez l'homme," Journal de pharmacie de Belgique, vol. 54, no. 3, pp. 75-82, 1999.

[38] K. Fujie and H. Ito, "Distribution and excretion of 1,3,5-trihydroxybenzene," Arzneimittelforschung, vol. 22, no. 4, pp. 777$780,1972$.

[39] M. Żebrowska-Gamdzyk, M. Maciejczyk, A. Zalewska, K. Guzińska-Ustymowicz, A. Tokajuk, and H. Car, "Whey protein concentrate WPC- 80 intensifies glycoconjugate catabolism and induces oxidative stress in the liver of rats," Nutrients, vol. 10, no. 9, article 1178, 2018.

[40] A. M. Gusdon, K. X. Song, and S. Qu, "Nonalcoholic fatty liver disease: pathogenesis and therapeutics from a mitochondriacentric perspective," Oxidative Medicine and Cellular Longevity, vol. 2014, Article ID 637027, 20 pages, 2014.

[41] B. Quéguineur, L. Goya, S. Ramos, M. A. Martín, R. Mateos, and L. Bravo, "Phloroglucinol: antioxidant properties and effects on cellular oxidative markers in human HepG2 cell line," Food and Chemical Toxicology, vol. 50, no. 8, pp. 28862893, 2012.

[42] V. Mendes, R. Vilaça, V. De Freitas, P. M. Ferreira, N. Mateus, and V. Costa, "Effect of myricetin, pyrogallol, and phloroglucinol on yeast resistance to oxidative stress," Oxidative Medicine and Cellular Longevity, vol. 2015, Article ID 782504, 10 pages, 2015.

[43] K. A. Kang, K. H. Lee, S. Chae et al., "Cytoprotective effect of phloroglucinol on oxidative stress induced cell damage via catalase activation," Journal of Cellular Biochemistry, vol. 97, no. 3, pp. 609-620, 2006.

[44] S. Sahoo, D. N. Meijles, and P. J. Pagano, "NADPH oxidases: key modulators in aging and age-related cardiovascular diseases?," Clinical Science, vol. 130, no. 5, pp. 317-335, 2016.

[45] I. Grattagliano, G. Calamita, T. Cocco, D. Q. Wang, and P. Portincasa, "Pathogenic role of oxidative and nitrosative stress in primary biliary cirrhosis," World Journal of Gastroenterology, vol. 20, no. 19, pp. 5746-5759, 2014.

[46] A. Panic, J. Stanimirovic, M. Obradovic et al., "Estradiol-mediated regulation of hepatic iNOS in obese rats: impact of Src, ERK1/2, AMPK $\alpha$, and miR-221," Biotechnology and Applied Biochemistry, vol. 65, no. 6, pp. 797-806, 2018.

[47] M. Maciejczyk, J. Szulimowska, K. Taranta-Janusz, A. Wasilewska, and A. Zalewska, "Salivary gland dysfunction, protein glycooxidation and nitrosative stress in children with chronic kidney disease," Journal of Clinical Medicine, vol. 9, no. 5, article 1285, 2020.

[48] C. Gersch, S. P. Palii, W. Imaram et al., "Reactions of peroxynitrite with uric acid: formation of reactive intermediates, alkylated products and triuret, and in vivo production of triuret under conditions of oxidative stress," Nucleosides, Nucleotides and Nucleic Acids, vol. 28, no. 2, pp. 118-149, 2009.

[49] J. Toczewska, T. Konopka, A. Zalewska, and M. Maciejczyk, "Nitrosative stress biomarkers in the non-stimulated and stimulated saliva, as well as gingival crevicular fluid of patients with periodontitis: review and clinical study," Antioxidants, vol. 9, no. 3, p. 259, 2020.

[50] P. Pacher, J. S. Beckman, and L. Liaudet, "Nitric oxide and peroxynitrite in health and disease," Physiological Reviews, vol. 87, no. 1, pp. 315-424, 2007.

[51] K. Dhama, S. K. Latheef, M. Dadar et al., "Biomarkers in stress related diseases/disorders: diagnostic, prognostic, and thera- peutic values," Frontiers in Molecular Biosciences, vol. 6, p. 91, 2019.

[52] B. K. Tiwari, K. B. Pandey, A. B. Abidi, and S. I. Rizvi, "Markers of oxidative stress during diabetes mellitus," Journal of Biomarkers, vol. 2013, Article ID 378790, 8 pages, 2013.

[53] A. Klisic, A. Isakovic, G. Kocic et al., "Relationship between oxidative stress, inflammation and dyslipidemia with fatty liver index in patients with type 2 diabetes mellitus," Experimental and Clinical Endocrinology \& Diabetes, vol. 126, no. 6, pp. 371-378, 2018.

[54] J. Borys, M. Maciejczyk, B. Antonowicz et al., "Glutathione metabolism, mitochondria activity, and nitrosative stress in patients treated for mandible fractures," Journal of Clinical Medicine, vol. 8, no. 1, p. 127, 2019.

[55] T. M. Dreaden Kasson, S. Rexroth, and B. A. Barry, "Lightinduced oxidative stress, $\mathrm{N}$-formylkynurenine, and oxygenic photosynthesis," PLoS One, vol. 7, no. 7, article e42220, 2012.

[56] Y. K. Al-Hilaly, T. L. Williams, M. Stewart-Parker et al., "A central role for dityrosine crosslinking of amyloid- $\beta$ in Alzheimer's disease," Acta Neuropathologica Communications, vol. 2, no. 1, 2014.

[57] Z. Chen, R. Tian, Z. She, J. Cai, and H. Li, "Role of oxidative stress in the pathogenesis of nonalcoholic fatty liver disease," Free Radical Biology and Medicine, vol. 152, pp. 116-141, 2020.

[58] D. D. Xiong, M. Zhang, N. Li, J. F. Gai, L. Mao, and M. Li, "Mediation of inflammation, obesity and fatty liver disease by advanced glycation endoproducts," European Review for Medical and Pharmacological Sciences, vol. 21, no. 22, pp. 5172-5178, 2017.

[59] J. M. Kim, I.-H. Hwang, I.-S. Jang et al., “Houttuynia cordata Thunb promotes activation of HIF-1A-FOXO3 and MEF2A pathways to induce apoptosis in human HepG2 hepatocellular carcinoma cells," Integrative Cancer Therapies, vol. 16, no. 3, pp. 360-372, 2017.

[60] K. McArthur, L. W. Whitehead, J. M. Heddleston et al., "BAK/BAX macropores facilitate mitochondrial herniation and mtDNA efflux during apoptosis," Science, vol. 359, no. 6378, article eaao6047, 2018.

[61] Y. Y. Huang, A. C. H. Chen, J. D. Carroll, and M. R. Hamblin, "Biphasic dose response in low level lightherapy," DoseResponse, vol. 7, no. 4, pp. 358-383, 2009.

[62] Y. G. Figueroa, A. K. Chan, R. Ibrahim et al., "NF- $\kappa$ B plays a key role in hypoxia-inducible factor-1-regulated erythropoietin gene expression," Experimental Hematology, vol. 30, no. 12, pp. 1419-1427, 2002.

[63] M. J. Piao, M. J. Ahn, K. A. Kang et al., "Phloroglucinol inhibits ultraviolet $\mathrm{B}$ radiation-induced oxidative stress in the mouse skin," International Journal of Radiation Biology, vol. 90, no. 10, pp. 928-935, 2014.

[64] S. K. Goswami, S. K. Gangadarappa, M. Vishwanath et al., "Antioxidant potential and ability of phloroglucinol to decrease formation of advanced glycation end products increase efficacy of sildenafil in diabetes-induced sexual dysfunction of rats," Sexual Medicine, vol. 4, no. 2, pp. e106e114, 2016.

[65] E. Moine, P. Brabet, L. Guillou, T. Durand, J. Vercauteren, and C. Crauste, "New lipophenol antioxidants reduce oxidative damage in retina pigment epithelial cells," Antioxidants, vol. 7, no. 12, p. 197, 2018.

[66] J. Mária and Ž. Ingrid, "Effects of bioactive compounds on senescence and components of senescence associated secretory 
phenotypes in vitro," Food and Function, vol. 8, no. 7, pp. 2394-2418, 2017.

[67] E. J. Yang, S. Ahn, J. Ryu et al., "Phloroglucinol attenuates the cognitive deficits of the 5XFAD mouse model of Alzheimer's disease," PLoS One, vol. 10, no. 8, article e0135686, 2015.

[68] K. Broadley, L. Larsen, P. M. Herst, R. A. J. Smith, M. V. Berridge, and M. J. McConnell, "The novel phloroglucinol PMT7 kills glycolytic cancer cells by blocking autophagy and sensitizing to nutrient stress," Journal of Cellular Biochemistry, vol. 112, no. 7, pp. 1869-1879, 2011.

[69] T. Charytoniuk, N. Iłowska, K. Berk, K. Drygalski, A. Chabowski, and K. Konstantynowicz-Nowicka, "The effect of enterolactone on sphingolipid pathway and hepatic insulin resistance development in HepG2 cells," Life Sciences, vol. 217, pp. 1-7, 2019.

[70] M. Maciejczyk, K. Taranta-Janusz, A. Wasilewska, A. Kossakowska, and A. Zalewska, "A case-control study of salivary redox homeostasis in hypertensive children. Can salivary uric acid be a marker of hypertension?," Journal of Clinical Medicine, vol. 9, no. 3, p. 837, 2020.

[71] H. P. Misra and I. Fridovich, "The role of superoxide anion in the autoxidation of epinephrine and a simple assay for superoxide dismutase," Journal of Biological Chemistry, vol. 247, no. 10 , pp. 3170-3175, 1972.

[72] "Oxygen radicals in biological systems, volume 105 - 1st edition,” December 2020, https://www.elsevier.com/books/ oxygen-radicals-in-biological-systems/kaplan/978-0-12182005-3.

[73] D. E. Paglia and W. N. Valentine, "Studies on the quantitative and qualitative characterization of erythrocyte glutathione peroxidase," The Journal of Laboratory and Clinical Medicine, vol. 70, no. 1, pp. 158-169, 1967.

[74] C. E. Mize and R. G. Langdon, "Hepatic glutathione reductase. I. Purification and general kinetic properties," The Journal of Biological Chemistry, vol. 237, pp. 1589-1595, 1962.

[75] O. W. Griffith, "Determination of glutathione and glutathione disulfide using glutathione reductase and 2-vinylpyridine," Analytical Biochemistry, vol. 106, no. 1, pp. 207-212, 1980.

[76] B. Choromańska, P. Myśliwiec, M. Łuba et al., “A longitudinal study of the antioxidant barrier and oxidative stress in morbidly obese patients after bariatric surgery. Does the metabolic syndrome affect the redox homeostasis of obese people?," Journal of Clinical Medicine, vol. 9, no. 4, p. 976, 2020.

[77] K. K. Griendling, C. A. Minieri, J. D. Ollerenshaw, and R. W. Alexander, "Angiotensin II stimulates NADH and NADPH oxidase activity in cultured vascular smooth muscle cells," Circulation Research, vol. 74, no. 6, pp. 1141-1148, 1994.

[78] J. Borys, M. Maciejczyk, B. Antonowicz, J. Sidun, M. Świderska, and A. Zalewska, "Free radical production, inflammation and apoptosis in patients treated with titanium mandibular fixations - an observational study," Frontiers in Immunology, vol. 10, 2019.

[79] N. Prajda and G. Weber, "Malignant transformation-linked imbalance: decreased xanthine oxidase activity in hepatomas," FEBS Letters, vol. 59, no. 2, pp. 245-249, 1975.

[80] M. B. Grisham, G. G. Johnson, and J. R. Lancaster, "Quantitation of nitrate and nitrite in extracellular fluids," Methods in Enzymology, vol. 268, pp. 237-246, 1996.

[81] J. S. Beckman, H. Ischiropoulos, L. Zhu et al., "Kinetics of superoxide dismutase- and iron-catalyzed nitration of pheno- lics by peroxynitrite," Archives of Biochemistry and Biophysics, vol. 298, no. 2, pp. 438-445, 1992.

[82] K. Grintzalis, D. Zisimopoulos, T. Grune, D. Weber, and C. D. Georgiou, "Method for the simultaneous determination of free/protein malondialdehyde and lipid/protein hydroperoxides," Free Radical Biology and Medicine, vol. 59, pp. 27-35, 2013.

[83] J. A. Buege and S. D. Aust, "Microsomal lipid peroxidation," Methods in Enzymology, vol. 52, no. C, pp. 302-310, 1978.

[84] M. Kalousová, T. Zima, V. Tesař, and J. Lachmanová, "Advanced glycation end products and advanced oxidation protein products in hemodialyzed patients," Blood Purification, vol. 20, no. 6, pp. 531-536, 2002. 